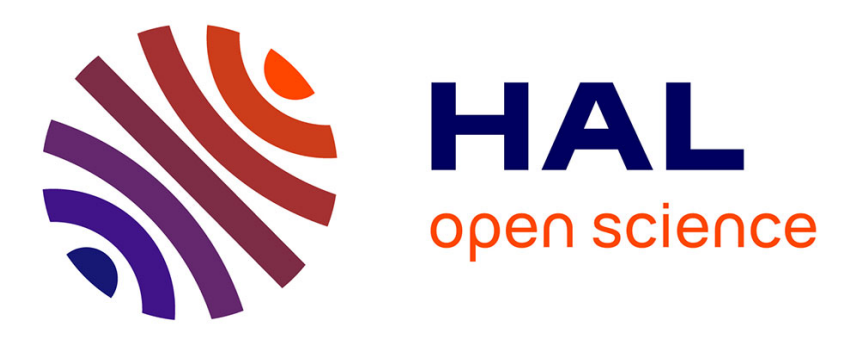

\title{
Trajectoire d'évolution d'un cours d'eau à très faible énergie: le cas de la Charente entre Angoulême et Saintes (Ouest de la France)
}

Amélie Duquesne, Christine Plumejeaud-Perreau, Jean-Michel Carozza

\section{To cite this version:}

Amélie Duquesne, Christine Plumejeaud-Perreau, Jean-Michel Carozza. Trajectoire d'évolution d'un cours d'eau à très faible énergie: le cas de la Charente entre Angoulême et Saintes (Ouest de la France). Géomorphologie: relief, processus, environnement, 2020, 26 (2), 10.4000/geomorphologie.14411 . hal-02615143

\section{HAL Id: hal-02615143 \\ https://hal.science/hal-02615143}

Submitted on 15 Jul 2020

HAL is a multi-disciplinary open access archive for the deposit and dissemination of scientific research documents, whether they are published or not. The documents may come from teaching and research institutions in France or abroad, or from public or private research centers.
L'archive ouverte pluridisciplinaire HAL, est destinée au dépôt et à la diffusion de documents scientifiques de niveau recherche, publiés ou non, émanant des établissements d'enseignement et de recherche français ou étrangers, des laboratoires publics ou privés. 
Géomorphologie : relief, processus,

Trajectoire d'évolution d'un cours d'eau à très faible énergie : le cas de la Charente entre Angoulême et Saintes (Ouest de la France)

Amélie Duquesne, Christine Plumejeaud-Perreau et Jean-Michel Carozza

\section{(2) OpenEdition}

Édition électronique

URL : http://journals.openedition.org/geomorphologie/14411

ISSN : 1957-777X

Éditeur

Groupe français de géomorphologie

\section{Référence électronique}

Amélie Duquesne, Christine Plumejeaud-Perreau et Jean-Michel Carozza, «Trajectoire d'évolution d'un cours d'eau à très faible énergie : le cas de la Charente entre Angoulême et Saintes (Ouest de la

France) », Géomorphologie : relief, processus, environnement [En ligne], Articles sous presse, mis en ligne le 02 mai 2020, consulté le 04 mai 2020. URL : http://journals.openedition.org/geomorphologie/14411 


\title{
Trajectoire d'évolution d'un cours d'eau à très faible énergie : le cas de la Charente entre Angoulême et Saintes (Ouest de la France)
}

\section{Trajectory of change of a low-energy river: the case of the Charente River between Angoulême and Saintes (West of France)}

\author{
Amélie Duquesne a*, Christine Plumejeaud-Perreau ${ }^{a}$, Jean-Michel Carozza ${ }^{a}$ \\ ${ }^{a}$ La Rochelle Université, LIENSs UMR 7266 CNRS - 2 rue Olympe de Gouges, 17 000, La Rochelle, France.
}

INFORMATION SUR L'ARTICLE

Reçu le 04 novembre 2019.

Reçu sous sa forme révisée le 03 mars 2020

Définitivement accepté le 21 avril 2020

${ }^{*}$ Auteur correspondant. Tél : $+33(0) 546458274$

Courriels : amelie.duquesne1@univ-lr.fr

(A. Duquesne)

christine.plumejeaud-perreau@univ-lr.f

(C. Plumejeaud-Perreau)

jean-michel.carozza@univ-lr.fr (J.-M. Carozza)

\begin{abstract}
RÉSUMÉ
Les connaissances sur l'évolution historique de la dynamique des cours d'eau à faible énergie et peu mobiles sont fondamentales pour anticiper la réponse de ces systèmes fluviaux aux interventions de gestion et de restauration. Toutefois, les ajustements de ce type de cours d'eau restent peu documentés. Cette étude analyse l'évolution historique de la moyenne Charente (segment fluvial Angoulême-Saintes), cours d'eau à faible énergie et peu mobile, sur les 250 dernières années, et adapte une méthode géomorphométrique jusque-là appliquée principalement sur des cours d'eau à moyenne et forte énergie où les ajustements fluviaux sont plus observables. Cette méthode s'appuie sur une approche quantitative basée sur le calcul d’indices géomorphométriques réalisés à intervalle régulier le long du linéaire étudié sur quatre cartes historiques (1866, 1899, 1972 et 2017). Les données géomorphométriques font l'objet d'une analyse factorielle par Analyse en Composantes Principales, d'une Classification Ascendante Hiérarchique et d'une segmentation par détection automatique de ruptures spatiales sous le logiciel R. Les résultats montrent une stabilité des îles et des chenaux sur les deux derniers siècles qui résulte de la conjonction entre la faible énergie du fleuve et la forte cohésion des berges limoneuses. Cette stabilité est rompue, localement, par les interventions anthropiques. Lévolution de la Charente serait principalement commandée par la navigation et les pratiques d'entretien du chenal et des îles. Le manque de données géohistoriques et les limites de la méthode statistique ne qualifient ni ne quantifient les autres facteurs de contrôle anthropiques dans un fleuve aménagé essentiellement pour la navigation. Mots clés : fleuve, Charente, dynamique fluviale, anastomose, faible énergie, cartographique diachronique.
\end{abstract}

\section{ABSTRACT}

Knowledge of the historic trajectory of low-energy and low mobile river dynamic is fundamental key in anticipating the response of fluvial systems to management and restoration interventions. However, studies on channel changes on this type of rivers are scarce. This study analyzes the historic trajectory of changes of the middle Charentre River (fluvial segment Angoulême-Saintes), low-energy and low mobile river, over a 250 years period, and to adapt the geomorphometric method applied so far on moderate to high energy rivers. This quantitative method is based on the calculation of geomorphometrical parameters at regularly spaced intervals along the study reach on four historic maps (1866, 1899, 1972 and 2017). Geomorphometrical data are analysed by Principal Component Analysis, Hierarchical Cluster Analysis and automatic spatial ruptures detection using the statistical package of $R$ software. Results show channels and islands stability over the last two centuries. This stability is interpreted as the combination of the low energy of the river and the high silty banks cohesion. It seems to be broken at local scale by human interventions. Most of observed changes on the Charente River seem controlled by navigation and maintenance of the channel and islands. The lack of geohistorical data and the limitations of statistic method do not qualify and quantify the other anthropogenic controlling factors in the river managed essentially for navigation.

Keywords: river, Charente River, fluvial dynamic, anastomosis, low-energy, diachronic mapping.

\section{Introduction}

Les connaissances sur l'évolution historique de la dynamique fluviale des cours d'eau sont fondamentales pour permettre d'anticiper la réponse des systèmes fluviaux dans la perspective du Changement Climatique (Mackay et al., 2005) ou de leur aménagement et désaménagement. Au cours des trente dernières années, les recherches sur les ajustements fluviaux anciens (i.e., transition Pléistocène-Holocène et intra-Holocène) et récents (XVIII ${ }^{\mathrm{e}}-\mathrm{XX}{ }^{\mathrm{e}}$ siècles) se sont multipliées. Ces travaux ont principalement porté sur les grands fleuves (Everitt, 1993 ; Porter et Massong, 2002 ; Pišút, 2002 ; Tiegs et Pohl, 2005 ; Swanson et al., 2010 ; Brunier et al., 2014 ; Joshi et Jun, 2017 ; Golovlyov et al., 2019) et les cours d'eau à moyenne et forte énergie (Bray, 1987 ; Hadley et Emmett, 1998 ; Winterbottom, 2000 ; Liébault et Piégay, 2002 ; Rinaldi, 2003 ; Keesstra et al., 2005 ; Surian et al., 2009 ; Zawiejska et Wyźga, 2010 ; Ollero, 2010 ; Aucelli et al., 2011 ; Perşoiu et Rădoane, 
2011 ; Comiti et al., 2011 ; Comiti, 2012 ; Kiss et Blanka, 2012 ; Rădoane et al., 2013 ; Ziliani et Surian, 2012 ; Scorpio et al., 2015 ; David et al., 2016).

Les cours d'eau à faible énergie ont suscité moins de recherches. Cependant, ces dernières années, l'attention portée à ce type de cours d'eau " ordinaires " a interrompu l'atonie relative de la recherche sur ce type de milieu (Lespez, 2012; Slowik, 2013 ; Lespez et al., 2015 ; Dépret et al., 2015 ; Slowik, 2015 ; Lespez et al., 2016 ; Corbonnois et al., 2016 ; Dépret et al., 2017 ; Beauchamp et al., 2017 ; Vayssière, 2018 ; Jugie et al., 2018 ; Vayssière et al., 2019 ; Slowik et al., 2020). Plusieurs de ces études se focalisent sur des petits cours d'eau de longueur réduite et dont le bassin versant est inférieur à $5000 \mathrm{~km}^{2}$. Ces cours d'eau présentent un style fluvial à méandre ou plus rarement à anastomose peu active en raison de la pente faible et d'un débit modeste qui impliquent des puissances spécifiques faibles $\left(<35 \mathrm{~W} \cdot \mathrm{m}^{-2}\right)$ et des difficultés à se réajuster suite aux aménagements du chenal ou des berges (Lespez, 2012). La compréhension de la réponse des cours d'eau à faible énergie, peu mobiles et à faible capacité d'ajustement, est un enjeu en termes de connaissance fondamentale de la dynamique fluviale dans un contexte où se multiplient les opérations de désaménagement sur ce type de rivière.

Ce travail analyse l'évolution du fleuve Charente, un cours d'eau à faible énergie, au cours des 250 dernières années. Jusqu'à présent, seuls quelques rares travaux documentent le fonctionnement hydrologique de ce cours d'eau (Pinard, 1965 ; Giret, 2002, 2004). Le fleuve Charente, pourtant le septième fleuve français par sa longueur, n'a, jusqu'à présent, fait l'objet d'aucune étude sur sa dynamique hydrogéomorphologique. Les enjeux de gestion quantitative des eaux et de gestion des étiages (ЕPTB Charente, 2004) et de restauration des continuités écologiques et sédimentaires du cours d'eau (EPTB Charente, 2003 ; Cellule Migrateurs Charente Seudre, 2018) s'appuient uniquement sur des connaissances empiriques.

D’après la récente classification de Gurnell et al. (2014) construite à partir de la classification de Nanson et Croke (1992), la Charente se rattache aux cours d'eau à très faible énergie de la façade atlantique (Candel, 2020) par sa puissance spécifique extrêmement faible (proche ou inférieure à $10 \mathrm{~W} \cdot \mathrm{m}^{-2}$,). Ce type de cours d'eau est également caractérisé par un débit de plein bord modeste, une pente très faible, une charge granulométrique très fine et cohésive d'où une migration latérale très limitée et par une plaine alluviale formée par aggradation verticale (dépôts de sédiments fins et avulsions occasionnelles) (Nanson et Croke, 1992). La Charente se singularise par la coexistence d'anastomoses complexes (Nanson et Croke, 1992 ; Carling et al., 2014) dans la partie supérieure de son linéaire entre Civray et Angoulême, d'anastomoses simples dans la partie médiane d'Angoulême à Cognac et d'un chenal unique méandriforme dans la partie inférieure de Cognac jusqu’à son embouchure estuarienne.

Ce travail cherche à reconstituer la trajectoire d'évolution de la Charente au cours des $\mathrm{XIX}^{\mathrm{e}}$ et $\mathrm{XX}^{\mathrm{e}}$ siècles et à proposer une interprétation des tendances évolutives observées. Il montre également l'intérêt et les difficultés à adapter une approche géomorphométrique pour étudier l'évolution des fleuves à faible énergie alors que, jusque-là, elle était principalement appliquée à des fleuves à mobilité importante (Gurnell et Downward, 1994 ; Gurnell, 1997 ; Uribelarrea et al., 2003 ; Nicoll et Hickin, 2010 ; Segura et Sanchis-Ibor, 2013 ; Toone et al., 2014 ; Scorpio et al.,
2018 ; Serlet et al., 2018). En raison des faibles vitesses d'évolution observées sur ce type de cours d'eau, il est parfois difficile de quantifier des ajustements fluviaux sur le temps long car les valeurs sont proches ou inférieures à l'incertitude générée par la méthode (e.g., erreur de données brutes, erreur de géoréférencement, erreur de digitalisation).

La dynamique fluviale de la Charente est appréhendée de manière classique par une approche quantitative basée sur la description géomorphométrique de la plaine alluviale et de son organisation. Cette description s'appuie sur une série d'indices quantitatifs (e.g., indice de sinuosité, largeur de la bande fluviale, etc.) mesurés/ calculés à intervalle régulier le long du linéaire d'étude pour 4 dates $(1866,1899,1972$ et 2017) sous Qgis et base de données spatiales PostGIS en adaptant la méthode proposée par David et al. (2016). Les données géomorphométriques font ensuite l'objet d'une analyse factorielle par Analyse en Composantes Principales (ACP) et d'une Classification Ascendante Hiérarchique (CAH). Les résultats permettent (i) une spatialisation des résultats obtenus par ACP et CAH en terme de typologie de dynamique du fond de vallée réalisée sous le logiciel $\mathrm{R}$; (ii) une segmentation du linéaire fluvial en zones homogènes à partir d'une détection automatique de ruptures spatiales faite sous le logiciel R ; (iii) une interprétation de la trajectoire d'évolution à l'échelle du linéaire et des trajectoires d'évolution à l'échelle de chaque zone, et (iv) le croisement de ces résultats avec les sources historiques (minutes, correspondances, rapports, affiches, plans, cartes postales, etc.) afin de les corréler avec les principaux forçages anthropiques à l'origine des modifications de trajectoire d'évolution à l'échelle du linéaire et des tronçons fluviaux.

\section{Zone d'étude}

La Charente draine un bassin versant de $10550 \mathrm{~km}^{2}$. Il s'étend sur les départements de la Dordogne, de la Haute-Vienne, de la Vienne, des Deux-Sèvres, de la Charente et de la Charente-Maritime (fig. 1). Il est composé de trois grands ensembles géologiques (Bry et Hoflack, 2004) : les formations sédimentaires calcaires du Jurassique au nord d'une ligne Angoulême-Rochefort ; au sud de cette dernière, les assises jurassiques sont recouvertes par des formations détritiques argilo-sableuses du Crétacé (EPTB Charente, 2016) ; enfin la partie amont est entaillée dans les complexes métamorphiques et granitiques du Massif Central. Les sols imperméables favorisent le ruissellement malgré un système de pentes faibles qui augmente le temps de ressuyage (EPTB Charente, 2016) à l'exception de la zone karstique de l'Angoumois (sous-bassins de la Tardoire, de la Bonnieure et de la Touvre).

Le bassin versant est caractérisé par un climat de type tempéré de façade océanique : doux et ensoleillé avec des précipitations modérées. Les précipitations annuelles varient entre 600 et $900 \mathrm{~mm}$, avec une moyenne de $800 \mathrm{~mm}$ (Bry et Hoflack, 2004). L'augmentation des précipitations s'effectue selon un gradient ouest-est en fonction de l'altitude, de 700 à $800 \mathrm{~mm}$ à Rochefort, et de 900 à $1000 \mathrm{~mm}$ à La Rochefoucauld (EPTB Charente, 2016).

Longue de $365 \mathrm{~km}$, la Charente draine un réseau hydrographique total de $6650 \mathrm{~km}$. Elle prend sa source à Chéronnac (département de la Vienne) à $295 \mathrm{~m}$ d'altitude. La pente moyenne est très faible, de l'ordre de $0,86 \%$. Elle est cependant plus forte en amont $(1,46 \%$ 


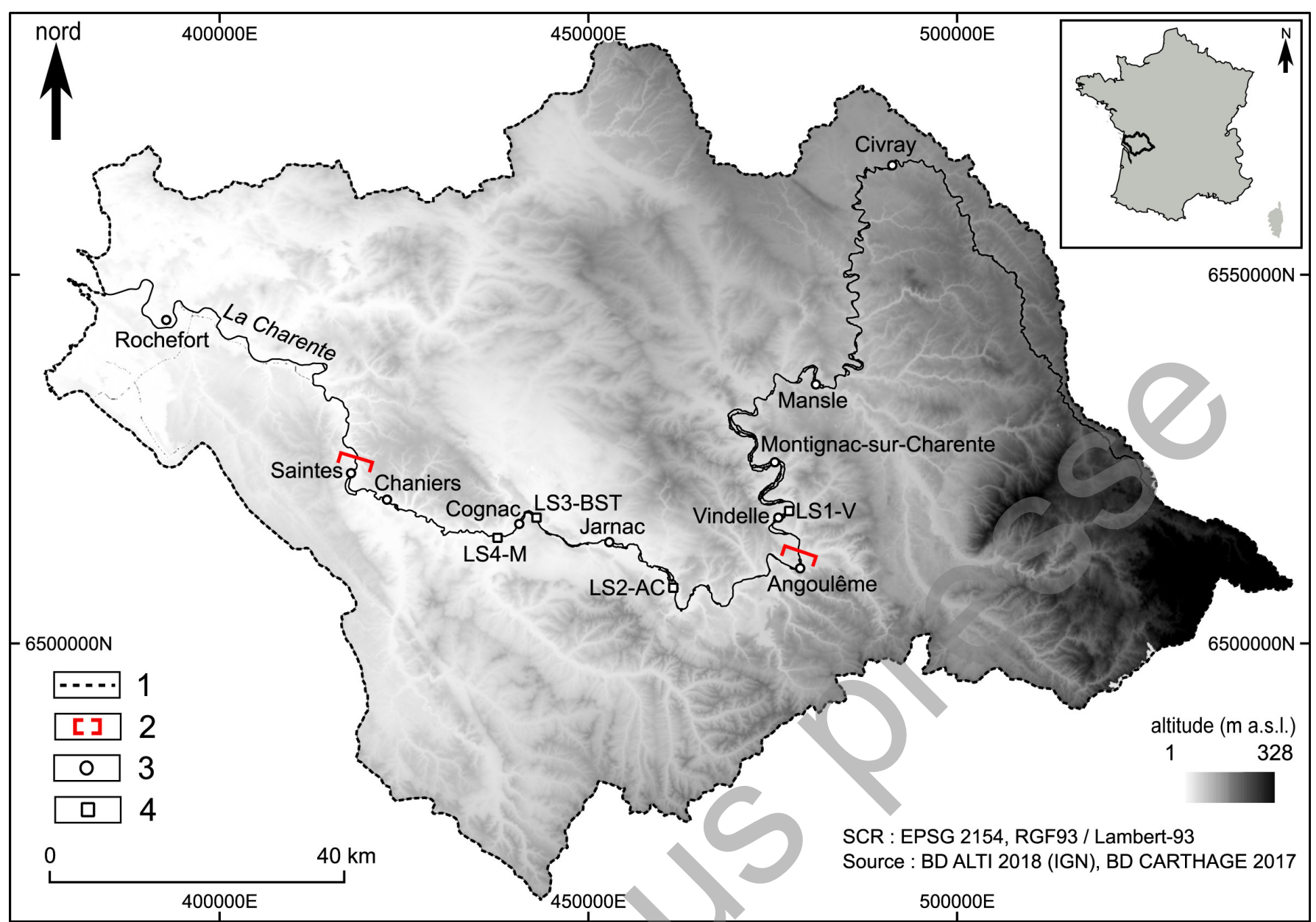

Fig. 1 - Localisation du bassin versant de la Charente.

1. Bassin versant de la Charente; 2 . Zone d'étude ; 3 . Agglomérations ; 4 . Logs sédimentaires.

Fig. 1 - Location of the catchment of the Charente River.

1. Catchment of the Charente River; 2. Study area; 3. Cities; 4. Sedimentary logs.

en amont de Montignac pour $142 \mathrm{~km})$, très faible dans sa partie médiane ( $0,42 \%$ dans l'Angoulêmois) et extrêmement faible dans sa partie aval jusqu'à Port-des-Barques (0,04\% pour les 100 derniers kilomètres) (EPTB Charente, 2003) (fig. 2).

A Saintes, le module de la Charente est de $140 \mathrm{~m}^{3} \cdot \mathrm{s}^{-1}$. Les débits mensuels moyens varient entre $80 \mathrm{~m}^{3} \cdot \mathrm{s}^{-1}$ aux basses eaux de fin d'été et $210 \mathrm{~m}^{3} \cdot \mathrm{s}^{-1}$ en janvier aux hautes eaux d'hiver (Grelie, 1933; Giret, 2002 ; EPTB Charente, 2016). La variabilité interannuelle est très élevée : le débit d'étiage peut s'abaisser jusqu'à $8 \mathrm{~m}^{3} \cdot \mathrm{s}^{-1}$ à Chaniers lors des étiages sévères (EPTB Charente, 2019) et à l'inverse les débits de crue atteignent $815 \mathrm{~m} \cdot \mathrm{s}^{-1}$ à Saintes comme lors de la crue centennale de 1982 (EPTB Charente, 2016). La faible capacité d'évacuation du lit mineur et la pente très faible sont à l'origine de débordements fréquents, importants et durables dans le lit majeur d'où l'identification du secteur d'étude comme un Territoire à Risque Important (TRI) d'inondation.

La faiblesse des valeurs de pente et de débit confère à la Charente une puissance spécifique très modeste (proche ou inférieure à $\left.10 \mathrm{~W} \cdot \mathrm{m}^{-2}\right)$. Cette dernière est faible dans la partie supérieure et médiane de la Charente, à Vindelle et à Jarnac, elle atteint $10 \mathrm{~W} \cdot \mathrm{m}^{-2}$,

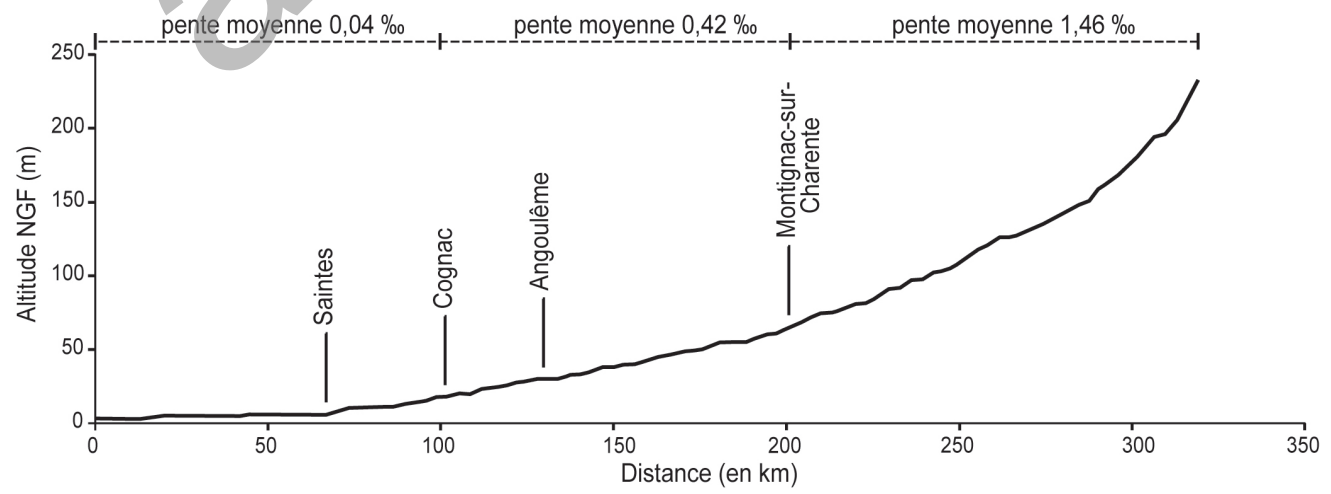

Fig. 2 - Profil en long de la Charente (Source : EPTB Charente, 2012).

Fig. 2 - Long profile of the Charente River (Source: EPTB Charente, 2012). 
et très faible dans la partie en aval, environ $7 \mathrm{~W} . \mathrm{m}^{-2}$ à Saintes. Les transports en suspension et de fond restent mal connus. Dabrin (2009) estime le flux particulaire de la Charente en baie d'Oléron à 78000 t.an $^{-1}$. Les premières constatations sur la charge de fond de la Charente montrent que les limons et les argiles sont dominants, la charge sableuse semblant être très limitée en aval de Jarnac. Ainsi, les berges, à l'image de la plaine alluviale, sont cohésives et stables car constituées essentiellement de limons et d'argiles (fig. 3).

Ce travail se concentre sur la moyenne Charente entre Angoulême et Saintes, un linéaire fluvial d'environ $90 \mathrm{~km}$ orienté est-ouest. Ce secteur est d'un intérêt particulier car la Charente passe d'un style fluvial anastomosé entre Angoulême et Cognac à un style à chenal unique méandriforme entre Cognac et Saintes. La Charente entre Angoulême et Cognac est constituée d'un chenal large, peu profond et rarement sinueux et de bras secondaires multiples, étroits et peu sinueux séparés par des îles de taille variable, enherbées et / ou boisées, allongées et peu larges. La plaine alluviale et les îles de grande dimension sont parcourues de paléochenaux longs, étroits et plus sinueux. La Charente entre Cognac et Saintes est quant à elle organisée en un chenal unique, large, peu profond inscrit dans une plaine alluviale plus étroite que dans la partie amont.

Le cours de la moyenne Charente a été fortement transformé par les actions anthropiques pour la navigation depuis le haut Moyen Âge (VIII ${ }^{\mathrm{e}}-\mathrm{IX}^{\mathrm{e}}$ siècles) (Dumont et Mariotti, 2013 ; Moyat, 2019 ;
Mariotti, 2019). Toutefois, les récentes découvertes archéologiques témoignent d'une navigation ancienne sur le fleuve au cours du Néolithique (6 000-2 200 BC), attestée par la découverte d'une pirogue monoxyle à Bourg-Charente (Billaud, 2010 ; Gailledreau, 2019), et de l'Antiquité (Deconinck, 2019), par la découverte de trois épaves romaines, deux en aval de Saintes, à Courbiac (Mariotti, 2018 ; Letuppe, 2019) ainsi qu’à Taillebourg (Rieth, 2010). Les interventions pour la navigation se sont accrues aux XVIII et XIX ${ }^{\text {e }}$ siècles (Jouannet, 1992 ; Renard, 1993). Trois grandes phases d'aménagement de la Charente se distinguent au cours de cette période : (i) les années 1775-1789 marquées par la construction de 21 écluses à sas, 8 en amont d'Angoulême et 13 en aval jusqu'à Jarnac, orchestrée par l'intendant Turgot et l'ingénieur en chef des Pontset-Chaussées Trésaguet dans le cadre de la politique d'amélioration de la navigabilité de la Charente; (ii) les années 1838 à 1859 voient la promulgation de la loi du 24 mars 1825 pour améliorer la navigation de la Charente : elle conduit à l'aménagement du secteur de la Baine à Chaniers (aménagement d'une dérivation, d'une écluse à sas et d'un barrage), à la construction d'un nouveau pont pour remplacer le pont gallo-romain à Saintes, au réaménagement des ports d'Angoulême, de Jarnac et de Cognac (quais et cales), au curage du chenal et à l'entretien des berges pour assurer la praticabilité du halage des gabarres; et (iii) la période 1881 à 1891 suite au décret présidentiel du 17 mars 1881 pour améliorer la navigation sur le département de

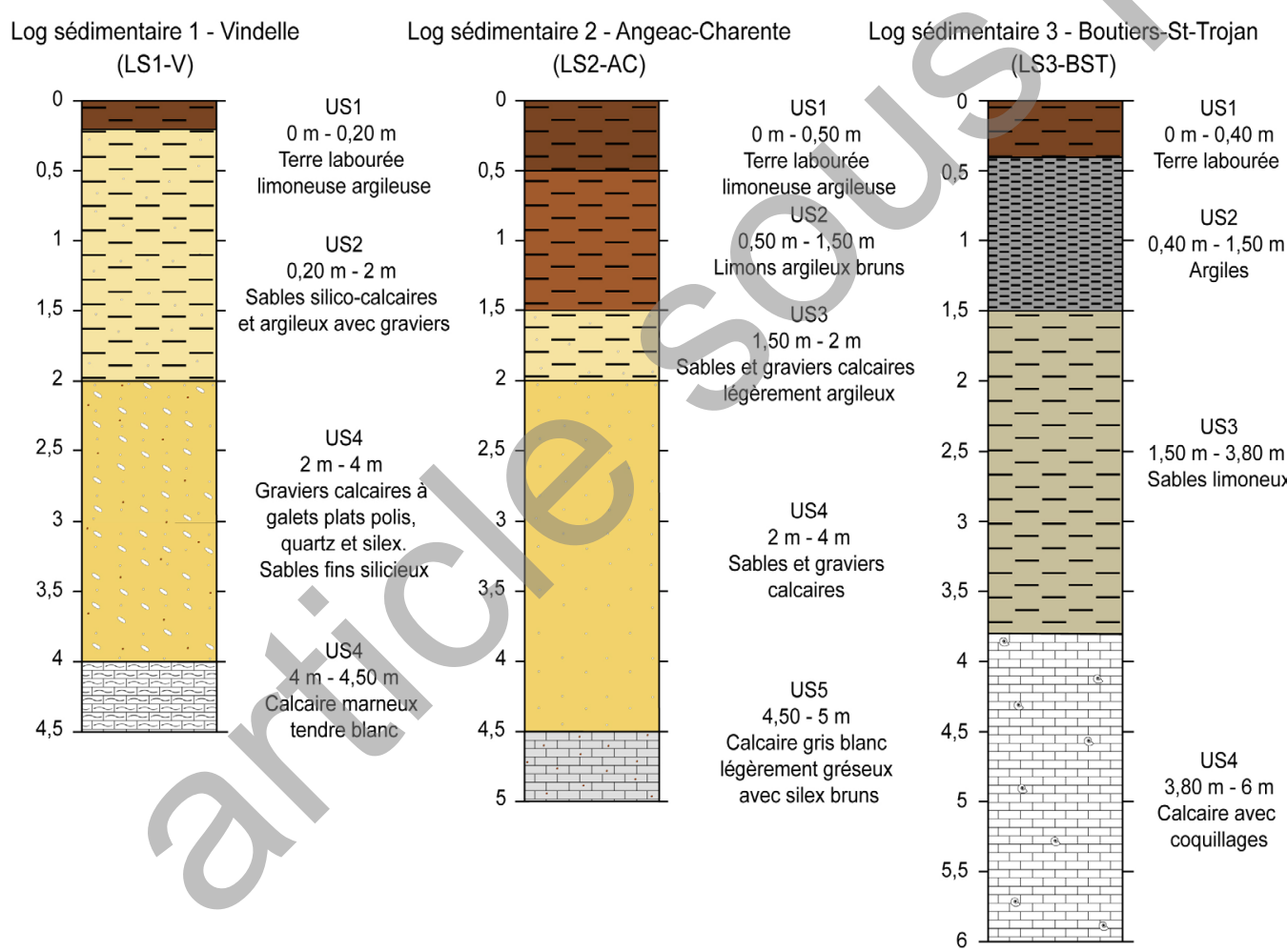

Fig. 3 - Logs stratigraphiques (LS1-V, LS2-AC, LS3-BST et LS4-M).

Source : Banque du sous-sol, BRGM.

Fig. 3 - Stratigraphic logs (LS1-V, LS2-AC, LS3-BST et LS4-M).

Source: Banque du sous-sol, BRGM.

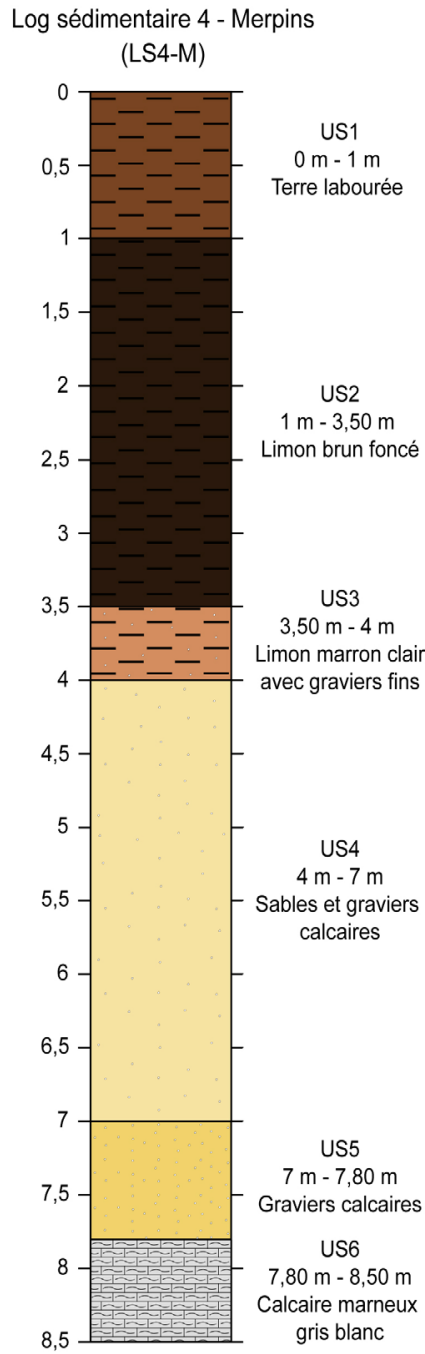


la Charente-Maritime : dragages du lit, réaménagement des zones portuaires à Saintes (aménagement de cales, reconstruction de quais, etc.) et réaménagement du barrage au secteur de la Baine à Chaniers.

La Charente a également été aménagée pour les besoins de l'exploitation de la force motrice de l'eau pour la mouture du blé depuis au moins le XVIII ${ }^{\mathrm{e}}$ siècle entre Angoulême et Saintes (13 moulins) (Jouannet, 1992) et pour la fabrication du papier depuis le $\mathrm{XVI}^{\mathrm{e}}$ siècle (66 moulins à papier en Angoumois en 1656) (Gaillard, 2018). Enfin, la Charente a aussi fait l'objet d'une exploitation des ressources halieutiques par les sociétés riveraines (pêcheries et anguillards). Les découvertes archéologiques témoignent de la présence de pêcheries depuis le Néolithique à Saint-Simon (Billaud, 2010 ; Gailledreau, 2019) et les sources écrites les mentionnent depuis le haut Moyen Âge (Jouannet, 1992). L'ensemble des moulins sur le linéaire fluvial entre Mansle et Saintes sont pourvus de pêcheries en 1778, 25 entre Mansle et Vars et 12 entre Angoulême et Cognac (Jouannet, 1992).

\section{Méthode}

\subsection{Constitution de la base de données cartographiques}

Au regard de la rareté des travaux antérieurs, ce travail a nécessité au préalable la constitution d'un corpus de données cartographiques inédit. Ces données proviennent du dépouillement des Archives Départementales de la Charente-Maritime et de la Charente : la
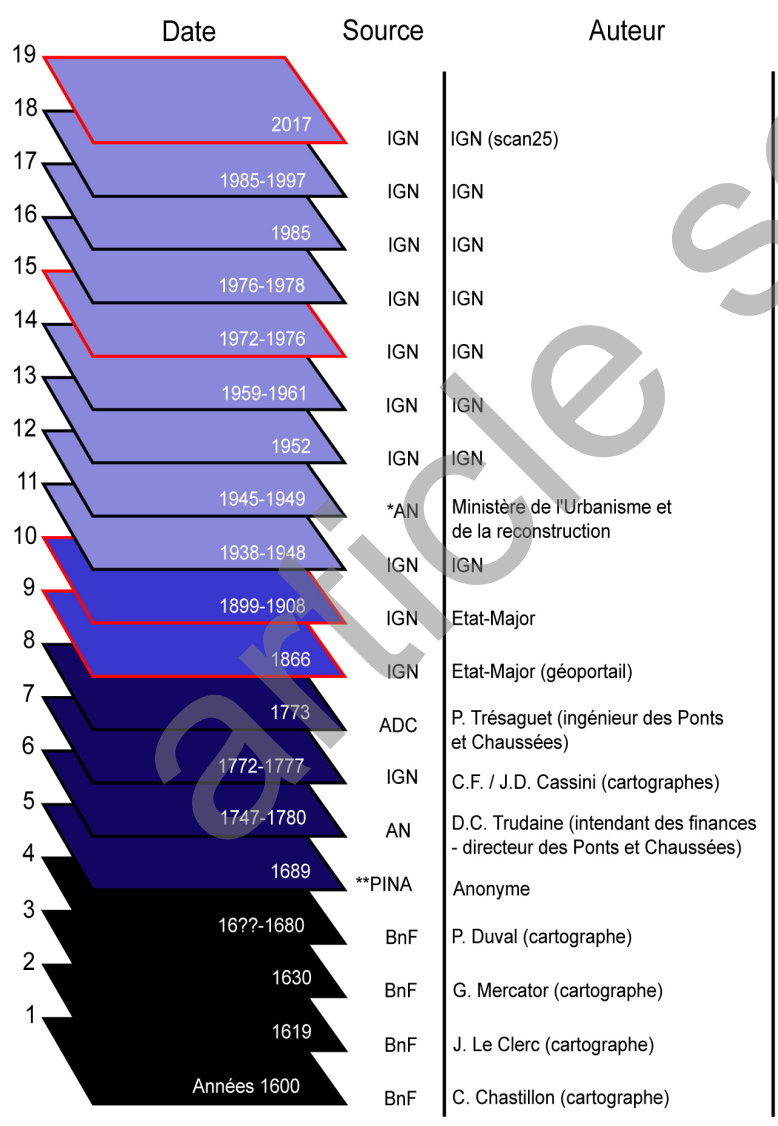

série S (travaux publics), la série W (archives contemporaines), la série Fi (fonds iconographique), la série L (archives révolutionnaires 1790-1800), la série K (lois, ordonnances, arrêtés depuis 1790) et la série 1C (fleuve Charente) spécifique aux Archives Départementales de la Charente. Elles ont été complétées par la consultation des fonds des Archives Municipales de Saintes et de Cognac, des fonds numérisés des Archives Nationales et des documents transmis par la cartothèque de l'Institut national de l'information géographique et forestière (IGN) et par le service Patrimoine et Inventaire de Nouvelle-Aquitaine. Le corpus de données cartographiques est présenté dans la Figure 4 . Il comporte 19 représentations totales ou partielles du fleuve Charente entre Angoulême et Saintes pour la période comprise entre 1600 et 2017. Ces dernières ont été réalisées à des fins de cartographie générale du territoire ou d’aménagements pour le péage ou pour l'amélioration de la navigation. La qualité des documents cartographiques est hétérogène et diminue généralement avec l'ancienneté en raison d'une usure des documents dans le temps, d'une échelle plus petite et des techniques topographiques utilisées lors des levés.

\subsection{Intégration des données géohistoriques dans un SIG}

\subsubsection{Géoréférencement des cartes}

En raison de l'imprécision des cartes anciennes des $\mathrm{XVII}^{e}$ et XVIH siècles, seules les cartes de 1866, 1899 (i.e., 1899 à 1908), 1972 (i.e., 1972 à 1976) et 2017 (Scan25) ont été intégrées dans un

\begin{tabular}{|c|c|c|}
\hline & Numérique & Totale \\
\hline & $\begin{array}{c}\text { Papier numérisé } \\
\text { Couleur }\end{array}$ & Totale \\
\hline 1:25000 & $\begin{array}{c}\text { Papier numérisé } \\
\text { Couleur }\end{array}$ & Totale \\
\hline 1:25000 & $\begin{array}{c}\text { Papier numérisé } \\
\text { Couleur }\end{array}$ & Totale \\
\hline $1: 50000$ & $\begin{array}{l}\text { Papier numérisé } \\
\text { Couleur }\end{array}$ & Totale \\
\hline $1: 25000$ & $\begin{array}{c}\text { Papier numérisé } \\
\text { Couleur }\end{array}$ & Totale \\
\hline 1:50 000 & $\begin{array}{l}\text { Papier numérisé } \\
\text { Couleur }\end{array}$ & Totale \\
\hline $\begin{array}{c}5000 / 1: 10000 \\
1: 20000\end{array}$ & $\begin{array}{c}\text { Papier numérisé } \\
\text { N\&B }\end{array}$ & Partielle \\
\hline 1:50 000 & $\begin{array}{c}\text { Papier numérisé } \\
\text { N\&B }\end{array}$ & Totale \\
\hline 1:50 000 & $\begin{array}{c}\text { Papier numérisé } \\
\text { N\&B }\end{array}$ & Totale \\
\hline $1: 40000$ & $\begin{array}{c}\text { Papier numérisé } \\
\text { Couleur }\end{array}$ & Totale \\
\hline$?$ & $\begin{array}{c}\text { Papier numérisé } \\
\text { Couleur }\end{array}$ & Partielle \\
\hline 1:86 400 & $\begin{array}{l}\text { Papier numérisé } \\
\text { Couleur }\end{array}$ & Totale \\
\hline $\begin{array}{c}8628 / 1: 8641 \\
1: 8660\end{array}$ & $\begin{array}{l}\text { Papier numérisé } \\
\text { Couleur }\end{array}$ & Anecdotique \\
\hline$?$ & $\begin{array}{l}\text { Papier numérisé } \\
\text { Couleur }\end{array}$ & Totale \\
\hline $20 \mathrm{~km}$ & $\begin{array}{c}\text { Papier numérisé } \\
\text { Couleur }\end{array}$ & Partielle \\
\hline $20 \mathrm{~km}$ & $\begin{array}{l}\text { Papier numérisé } \\
\text { Couleur }\end{array}$ & Totale \\
\hline $20 \mathrm{~km}$ & $\begin{array}{c}\text { Papier numérisé } \\
\text { Couleur }\end{array}$ & Totale \\
\hline$?$ & $\begin{array}{c}\text { Papier numérisé } \\
\text { N\&B }\end{array}$ & Anecdotique \\
\hline
\end{tabular}

\begin{tabular}{l}
\hline cartes topographiques IGN récentes \\
cartes d'Etat-Major des XIXe et XXe siècles \\
cartes du cours de la Charente des XVII et \\
XVIII ${ }^{\text {e }}$ siècles \\
cartes à vocation générale du XVII ${ }^{e}$ siècle \\
cartes retenues pour l'analyse quantitative \\
* documents transmis par les Archives \\
départementales de Charente \\
** documents transmis par Pascale Moisdon- \\
Pouvreau et Christine Sarrazin \\
ADC : Archives départementales de la Charente \\
AN : Archives nationales \\
BnF : Bibliothèque nationale de France \\
IGN : Institut national de l'information géographique \\
et forestière \\
PINA : Patrimoine et Inventaire de Nouvelle-Aquitain
\end{tabular}

PINA : Patrimoine et Inventaire de Nouvelle-Aquitaine

Fig. 4 - Inventaire des données cartographiques du fleuve Charente.

Fig. 4 - Inventory of historical maps of Charente River. 
SIG et une base de données spatiales PostGIS. Elles ont été projetées dans le système de projection cartographique EPSG :2154, RGF93/ Lambert-93 et ont fait l'objet d'un géoréférencement sous le logiciel Qgis 2.18.14 en prenant pour référentiel le Scan25, celui-ci étant déjà géoréférencé. Les points d'amers ont été répartis de part et d'autre $\mathrm{du}$ fleuve de manière homogène en utilisant principalement les intersections des routes afin d'obtenir un géoréférencement précis. Leur nombre varie entre 35 et 70 en fonction de l'ancienneté des cartes.

\subsubsection{Délimitation de la plaine alluviale}

La digitalisation de la plaine alluviale s'est faite sous le logiciel Qgis 2.18.14 à l'échelle 1/2 500. Les limites de la plaine alluviale ont été déterminées à partir de la carte géologique réalisée par le Bureau de recherches géologiques et minières (BRGM) et de la consultation des données de la Banque du sous-sol du BRGM. Elles ont été ensuite affinées par l'étude de la cartographie des zones inondées lors de la crue centennale de 1982 .

\subsubsection{Digitalisation des unités morphologiques}

À l'intérieur de la plaine alluviale, les chenaux, les îles et leur mode d'occupation du sol ont fait l'objet d'une digitalisation sous le logiciel Qgis 2.18.14 pour les cartes de 1866, 1899, 1972 et 2017. La digitalisation a été réalisée à l'échelle 1/2500 par le même opérateur afin d'éviter une hétérogénéité de l'interprétation des surfaces à digitaliser. Ces dernières sont de 4 types : (i) banc non-végétalisé, (ii) surface enherbée (i.e., herbes, arbustes), (iii) surface boisée (i.e., arbres), et (iv) surface en eau.

\subsection{Segmentation de la plaine alluviale}

La plaine alluviale a ensuite été subdivisée en tronçons de longueur fixe de $500 \mathrm{~m}$ sous le logiciel ArcGis suivant la méthode proposée par Albert et Piégay (2011) qui consiste à générer des polygones de Thiessen à partir de points localisés tous les $500 \mathrm{~m}$ le long de l'axe médian de la plaine alluviale (fig. 5A-B). Bien qu'elle permette de générer des polygones perpendiculaires à l'axe médian de la plaine alluviale, cette méthode présente l'inconvénient de créer des formes
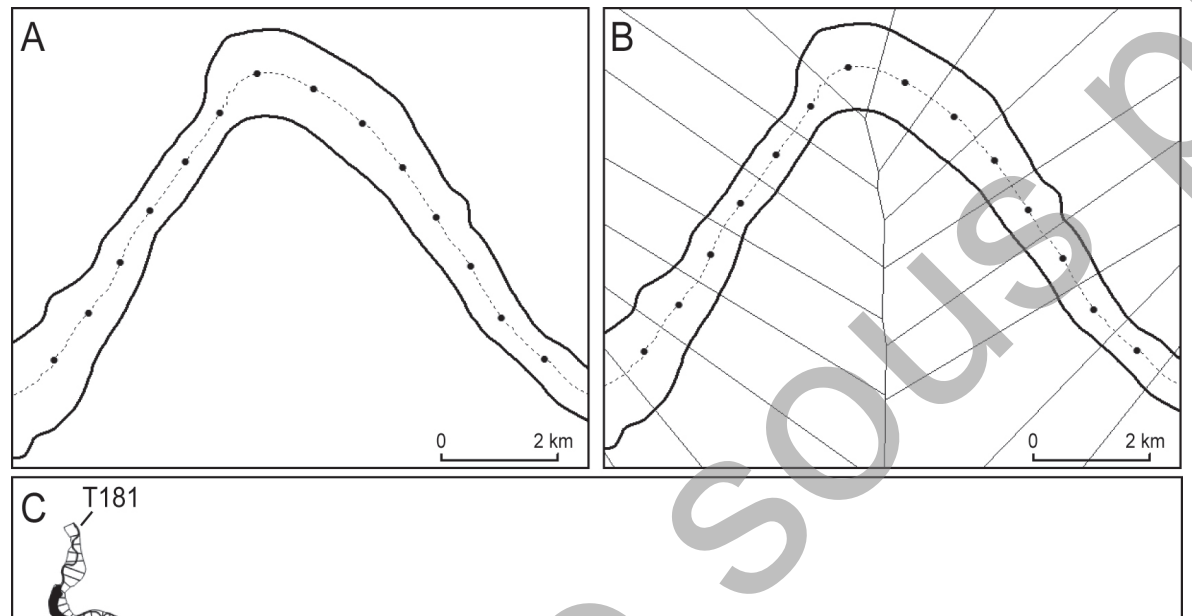

o
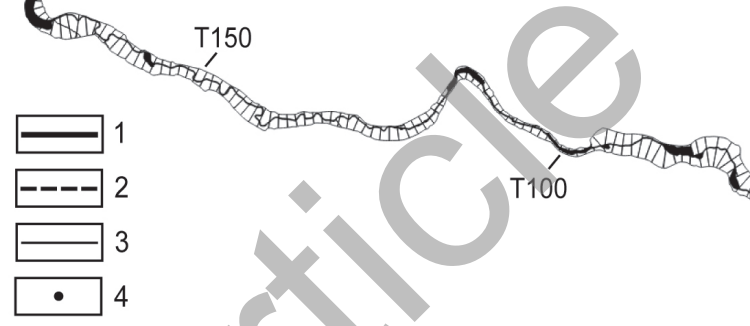

hétérogènes. Ainsi, la taille des tronçons de mesure peut différer dans les segments non rectilignes et, par conséquent, une extrémité du polygone est plus courte que l'autre. Cependant, l'impact de cette hétérogénéité reste limité. Au total, la plaine alluviale a été subdivisée en 181 tronçons numérotés de T001 à T181 de l'amont à l'aval (fig. 5C).

\subsection{Estimation des erreurs de mesure}

Malgré la mise en place de méthodes rigoureuses pour le géoréférencement et la digitalisation, les résultats obtenus incluent une part d'incertitude qu'il convient d'évaluer (Letortu, 2013). Trois sources d'erreurs sont identifiées (tab. 1) : (Depret, 2014).
Fig. 5 - Méthode utilisée pour la segmentation de la plaine alluviale de la Charente.

A : Délimitation de la plaine alluviale et génération de l’axe central de la plaine alluviale et des centroïdes des futurs tronçons ; B : Génération des polygones de Thiessen à partir de l'axe central de la plaine alluviale ; C : 181 tronçons de $500 \mathrm{~m}$ de longueur de la Charente. 1. Plaine alluviale; 2 . Axe central de la plaine alluviale ; 3 . Polygones de Thiessen non découpés ; 4 . Centroïdes des tronçons.

Fig. 5 - Method used for catchment of the alluvial plain of the Charente River.

A: Splitting of the alluvial plain and creation of the central line of the alluvial plain and centroids of coming units; B: Thiessen polygons generation from central line in the alluvial plain: C: Map of the 181 units of $500 \mathrm{~m}$ length of the Middle Charente River. 1. Alluvial plain; 2. Central line of alluvial plain; 3 . Thiessen polygons uncut; 4 . Centroids of units.

(i) l'erreur brute des données : elle est liée à la qualité intrinsèque des cartes. Elle dépend de la précision de la délimitation des objets à cartographier et de la précision de la méthode d'acquisition de ces objets. L'erreur est estimée à la taille du pixel.

(ii) la précision du géoréférencement : elle est déterminée par l'erreur quadratique moyenne (Root Mean Square Error ou RMSE) et correspond à la moyenne au carré de la différence en pixels entre les coordonnées d'un point d'amer sur le document de référence et celles de ce même point d'amer sur la carte. Elle est considérée comme une valeur proche et acceptable de l'erreur de rectification

(iii) l'erreur de digitalisation : elle correspond à l'incertitude maximale de localisation de la limite des formes fluviales. Cette 
incertitude repose sur la difficulté d'apprécier les limites ellesmêmes conditionnées par la résolution, l'échelle, la qualité des documents cartographiques et l'impression de l'opérateur (Thieler et Danforth, 1994a, 1994b ; Durand, 2000). L'erreur de digitalisation est évaluée à 1 pixel de largeur pour les cartes de bonne qualité et à
2 pixels de largeur pour les cartes de mauvaise qualité.

L'addition de ces trois types d'erreurs permet d'estimer la marge d'erreur globale pour chaque carte intégrée dans le SIG (tab. 1). Ces valeurs constituent des seuils à partir desquels une évolution est considérée comme significative (Faye et al., 2008).

Tab. 1 - Estimation de la marge d'erreur globale.

Tab. 1 - Estimation of the total margin of error.

\begin{tabular}{|c|c|c|c|c|c|c|c|c|}
\hline Carte & Année & $\begin{array}{l}\text { Référence } \\
\text { de la carte }\end{array}$ & $\begin{array}{l}\text { Taille du } \\
\text { pixel (m) }\end{array}$ & $\begin{array}{l}\text { Erreur brute } \\
\text { des données }\end{array}$ & $\begin{array}{c}\text { Erreur de } \\
\text { géoréférencement }\end{array}$ & $\begin{array}{c}\text { Erreur de } \\
\text { digitalisation }\end{array}$ & $\begin{array}{l}\text { Estimation } \\
\text { de l'erreur } \\
\text { globale }(\mathrm{m})\end{array}$ & $\begin{array}{c}\text { Erreur } \\
\text { quadratique } \\
\text { totale }(\mathrm{m})\end{array}$ \\
\hline \multirow{4}{*}{$\begin{array}{c}\text { Carte } \\
\text { d'Etat-Major }\end{array}$} & 1866 & IGNF Scan Saintes & 1,69 & $\pm 1,69$ & $\pm 36,11$ & $\pm 3,38$ & $\pm 41,18$ & 36,31 \\
\hline & 1866 & IGNF Scan Cognac & 1,69 & $\pm 1,69$ & $\pm 26,61$ & $\pm 3,38$ & $\pm 31,68$ & 26,88 \\
\hline & 1866 & $\begin{array}{c}\text { IGNF Scan } \\
\text { Bourg Charente }\end{array}$ & 1,69 & $\pm 1,69$ & $\pm 39,42$ & $\pm 3,38$ & $\pm 44,49$ & 39,60 \\
\hline & 1866 & $\begin{array}{l}\text { IGNF Scan } \\
\text { Angoulême }\end{array}$ & 1,69 & $\pm 1,69$ & $\pm 24,23$ & $\pm 3,38$ & & 24,52 \\
\hline \multirow{4}{*}{$\begin{array}{c}\text { Carte } \\
\text { d'Etat-Major } \\
(1899-1908)\end{array}$} & 1899 & $\begin{array}{c}\text { 5EM161 } \\
\text { N5_50k_1899 }\end{array}$ & 4,23 & $\pm 4,23$ & $\pm 12,60$ & $\pm 8,46$ & $\pm 25,29$ & 15,76 \\
\hline & 1908 & $\begin{array}{c}\text { 5EM162 } \\
\text { SO_50k_1908 }\end{array}$ & 4,23 & $\pm 4,23$ & $\pm 15,86$ & $\pm 8,46$ & $\pm 28,55$ & 18,47 \\
\hline & 1908 & $\begin{array}{c}\text { 5EM162 } \\
\text { NO_50k_1908 }\end{array}$ & 4,23 & $\pm 4,23$ & $\pm 15,09$ & & $\pm 27,78$ & 17,81 \\
\hline & 1908 & $\begin{array}{c}\text { 5EM162 } \\
\text { SE_50k_1908 }\end{array}$ & 4,24 & $\pm 4,24$ & $\pm 15,73$ & $\pm 8,48$ & $\pm 28,45$ & 18,37 \\
\hline \multirow{4}{*}{$\begin{array}{c}\text { Carte IGN } \\
(1972-1976)\end{array}$} & 1973 & 1531_50k_1973 & 4,23 & $\pm 4,23$ & $\pm 2,16$ & $\pm 4,23$ & $\pm 10,62$ & 6,36 \\
\hline & 1973 & 1532_50k_1973 & 4,23 & $\pm 4,23$ & $\pm 3,28$ & $\pm 4,23$ & $\pm 11,74$ & 6,82 \\
\hline & 1976 & 1632_50k_1976 & 4,23 & $\pm 4,23$ & $\pm 2,69$ & $\pm 4,23$ & $\pm 11,15$ & 6,56 \\
\hline & 1972 & 1732_50k_1972 & 4,23 & $\pm 4,23$ & $\pm 3,61$ & $\pm 4,23$ & $\pm 12,07$ & 6,99 \\
\hline Scan 25 & 2017 & $\begin{array}{c}\text { SCAN25 } \\
\text { Topographique L93 }\end{array}$ & 2,5 & $\pm 2,5$ & - & $\pm 2,5$ & \pm 5 & 3,54 \\
\hline
\end{tabular}

3.5. Réalisation d'une base de données géomorphométriques

Les quatre cartes font ensuite l'objet d'une analyse statistique à partir du calcul d'une série d'indices géomorphométriques descriptifs de la plaine alluviale, du chenal et des îles à l'échelle du linéaire et à l'échelle des tronçons de mesure. La méthode de calcul est présentée dans la Figure 6. Seize indices ont ainsi été mesurés afin d'estimer une évolution globale du fleuve Charente et de produire une typologie dynamique des formes fluviales et une segmentation du linéaire en unités à trajectoires homogènes sur la période 1866-2017.

\subsection{Analyse en Composantes Principales}

Dans ce travail, les données de niveau 3 (étude des évolutions temporelles multivariables et multi-individus) sont structurées par des tableaux de données ternaires $\mathrm{X}_{\mathrm{IIK}}$ (un cube de données). Ils croisent des objets I (les 181 tronçons fluviaux) sur lesquels des variables J (les 16 indices descriptifs) ont été mesurées ou renseignées aux différentes dates K (4 dates : 1866, 1899, 1972 et 2017). L'analyse de ces cubes de données est complexe mais l'Analyse en Composantes Principales (ACP) peut être envisagée comme une stratégie d'analyse (Pernin, 1986). Deux modalités simples peuvent être proposées pour la prise en compte de la dimension temporelle : l'analyse par dates séparées et l'analyse mélangée par dates (Tillard,
1996). Seule une modalité de l'analyse par dates séparées sera présentée dans le cadre de ce travail. D’autres méthodes statistiques plus complexes peuvent alors être développées. En raison de sa simplicité, cette méthode a par ailleurs été préférée à des méthodes factorielles plus complexes comme l'Analyse factorielle multiple mixte (AFMmix) (David et al., 2016).

\subsection{Classification Ascendante Hiérarchique}

La Classification Ascendante Hiérarchique $(\mathrm{CAH})$ a pour objectif d'élaborer une typologie des tronçons fluviaux. Elle recherche une homogénéité intra-classe et une hétérogénéité inter-classe c'est-àdire qu'elle regroupe les tronçons fluviaux les plus homogènes dans une même classe alors que les classes sont, elles, les plus dissemblables possibles. La CAH a été réalisée sous le logiciel $\mathrm{R}$ version 3.5.0 à l'aide du package Cluster avec la fonction agnes en utilisant la distance euclidienne entre les individus et la méthode d'agrégation de Ward. Trois classes ont ainsi été définies afin d'obtenir un effectif suffisant dans chacune d'entre elles et d'appliquer cette classification pour chaque année.

\subsection{Segmentation de la plaine alluviale de la Charente en unités homogènes}

Les trois classes générées à partir des quatre jeux de données sont considérées comme identiques aux quatre dates. Par conséquent, 


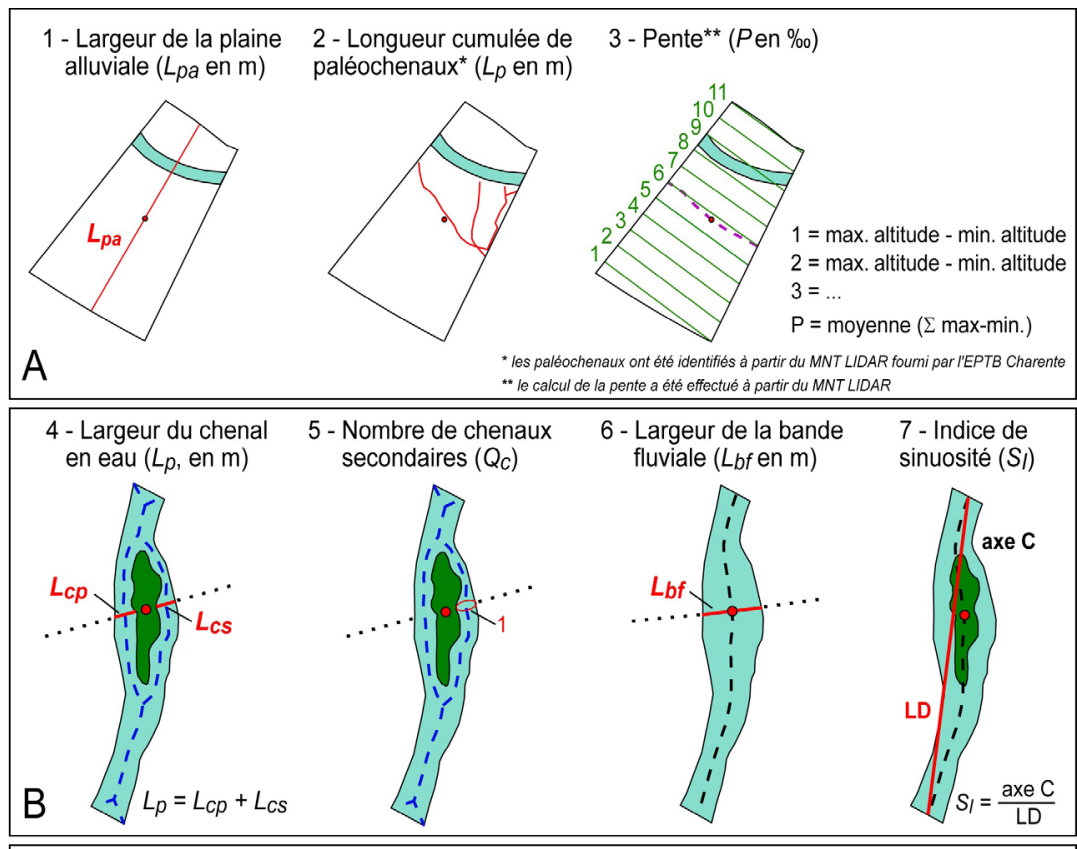

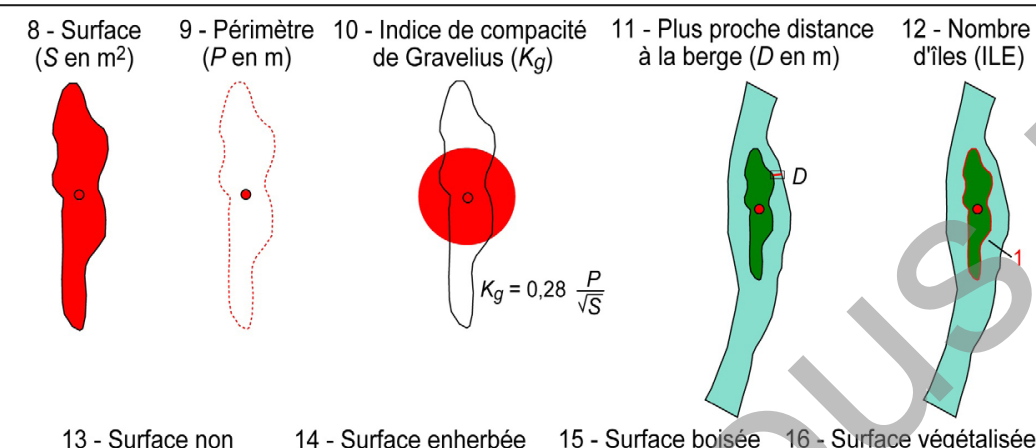
végétalisée $\left(S_{n v}\right.$ en $\left.\mathrm{m}^{2}\right)$

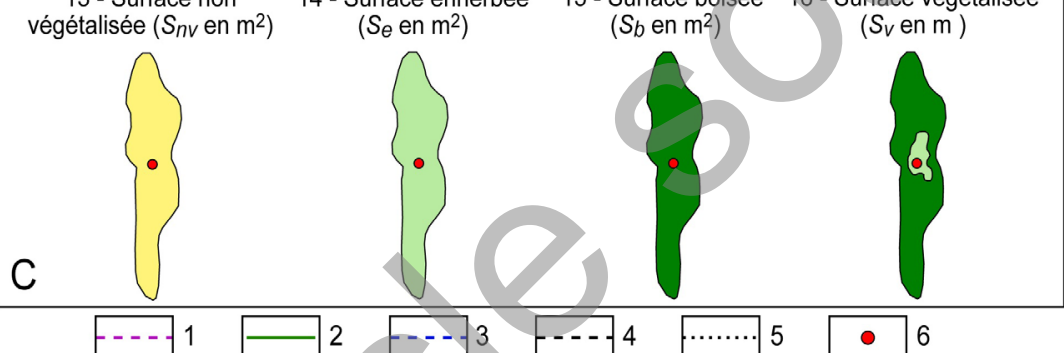

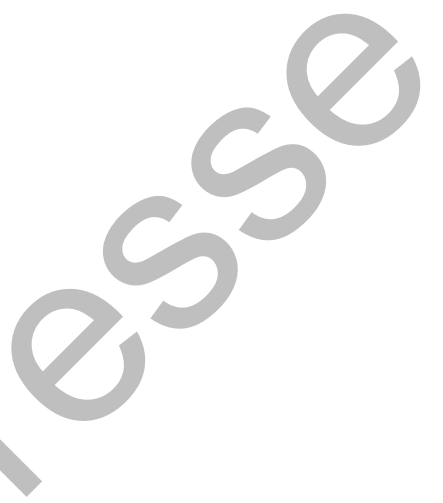

Fig. 6 - Indices géomorphométriques mesurés/ calculés à l'échelle des tronçons pour 1866, 1899, 1972 et 2017.

A : Indices géomorphométriques descriptifs de la plaine alluviale ; B : Indices géomorphométriques descriptifs du chenal; $\mathrm{C}$ : Indices géomorphométriques descriptifs des îles ; 1 . Axe central de la plaine alluviale; 2. Lignes parallèles à l'axe central de la plaine alluviale ; 3 . Axe central des chenaux ; 4 . Axe central du chenal; 5 . Lignes orthogonales; 6 . Centroïdes des tronçons ou des îles.

Fig. 6 - Geomorphometrical index measured/ calculated at the units scale for 1866, 1899, 1972 and 2017.

A: Geomorphometrical index of the alluvial plain; B: geomorphometrical index of the channel; $C$ : Geomorphometrical index of the islands; 1. Central line of alluvial plain; 2. Parallel lines to central line of alluvial plain; 3 . Central line of channels; 4 . Central line of channel; 5. Orthogonal lines; 6. Centroids of units or island. la spatialisation de cette typologie en trois classes permet de segmenter la plaine alluviale en unités homogènes d'un point de vue morphologique. Pour les quatre séries de données (1866, 1899, 1972 et 2017), une détection automatique de ruptures spatiales a été réalisée sous le logiciel $R$ version 3.5.0 à l'aide du package Changepoint.

\section{Résultats}

\subsection{Résultats de la Classification Ascendante Hiérarchique}

Après la réalisation de l'ACP et de la $\mathrm{CAH}$ pour chacun des quatre tableaux $\mathrm{X}_{\mathrm{k}}$, les ellipses et les centres de gravité des classes ont été projetés sur un plan factoriel (Faye et al., 1991). Le cercle de corrélation des variables et la projection des individus sur les deux premiers axes factoriels sont présentés sur les Figures 7 et 8 . La structure des données évolue peu entre les dates ce qui rend cette méthode graphique acceptable (i.e. quasi-stationnarité) (Casin et al., 2011). Bien que l'absence de repère commun mentionnée par Faye et al. (1991) limite la possibilité d'identifier clairement l'impact de chaque facteur de contrôle sur le cours d'eau, cela n'empêche pas pour autant de proposer des pistes de réflexion dans l'interprétation des résultats obtenus.

Trois classes ont été déterminées par la CAH. Elles sont décrites ici à l'aide des variables de 2017 :

(i) Les tronçons fluviaux de la classe 1 se distinguent par une bande fluviale large (moy. $157 \mathrm{~m}$ ), un chenal en eau principal large (moy. $87 \mathrm{~m}$ ), la présence de chenaux secondaires (max. 5 chenaux) et un nombre important d'îles enherbées ou boisées (max. 5 îles). Les îles sont de taille réduite (moy. $15486 \mathrm{~m}^{2}$ ) et de forme allongée (moy. 1,40 m). La plaine alluviale est quant à elle plus étroite (moy. 690 m).

(ii) La classe 2 rassemble les tronçons fluviaux caractérisés par un style à chenal unique méandriforme. Ils sont déterminés par une bande fluviale (moy. $62 \mathrm{~m}$ ) et une plaine alluviale réduites (moy. 


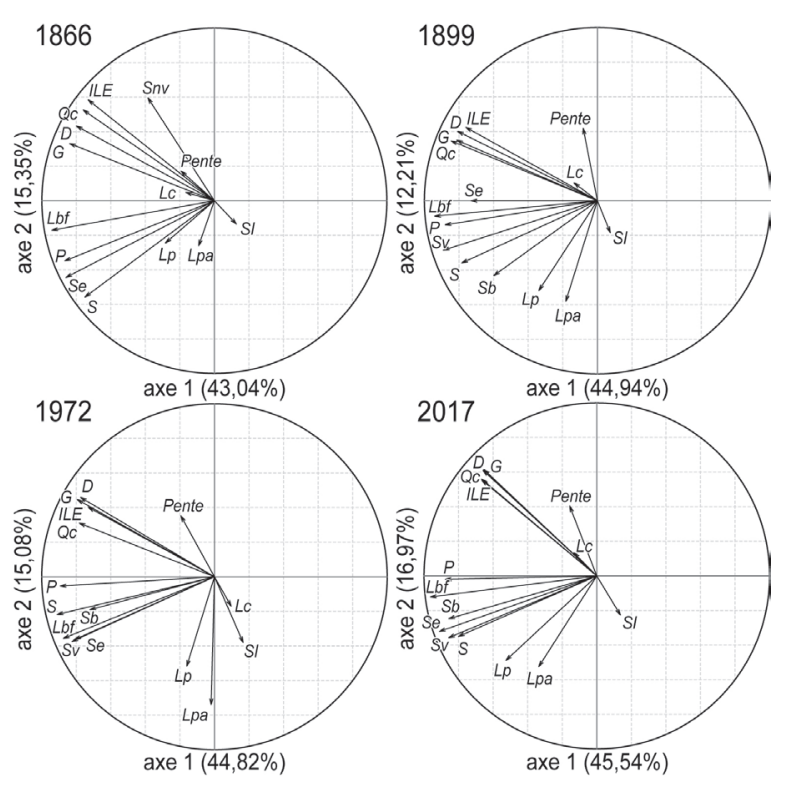

Fig. 7 - Cercle de corrélation des variables (1866, 1899, 1972 et 2017).

Fig. 7 - Circle of correlation (1866, 1899, 1972 et 2017).

$811 \mathrm{~m}$ ), un chenal en eau principal plus étroit (moy. $75 \mathrm{~m}$ ) et la quasiabsence de paléo-chenaux (moy. $256 \mathrm{~m}$ ).

(iii) La classe 3 regroupe les tronçons fluviaux marqués par une bande fluviale très large (moy. 530,57 m), un chenal en eau principal large (moy. $97 \mathrm{~m}$ ) et un nombre réduit de chenaux secondaires (moy. 2 chenaux). Ces tronçons sont caractérisés par la présence de grandes îles végétalisées (moy. $141249 \mathrm{~m}^{2}$ ). Elles sont larges, de forme allongée (moy. $1399 \mathrm{~m}$ ) et relativement proches de la berge (min. $6 \mathrm{~m}$ ). La plaine alluviale est très étendue (moy. $1222 \mathrm{~m}$ ) et est parcourue par de très longs paléo-chenaux (moy. $1295 \mathrm{~m}$ et max. $4464 \mathrm{~m})$.
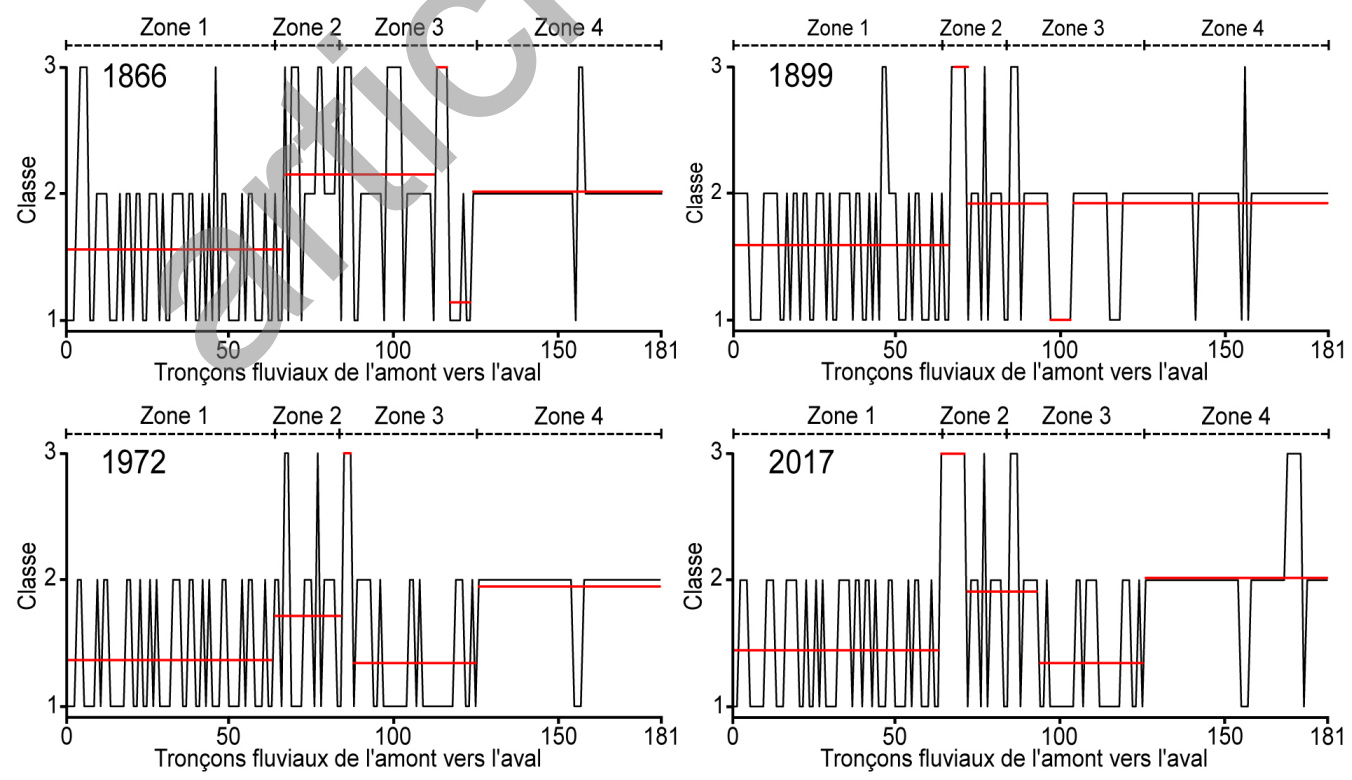

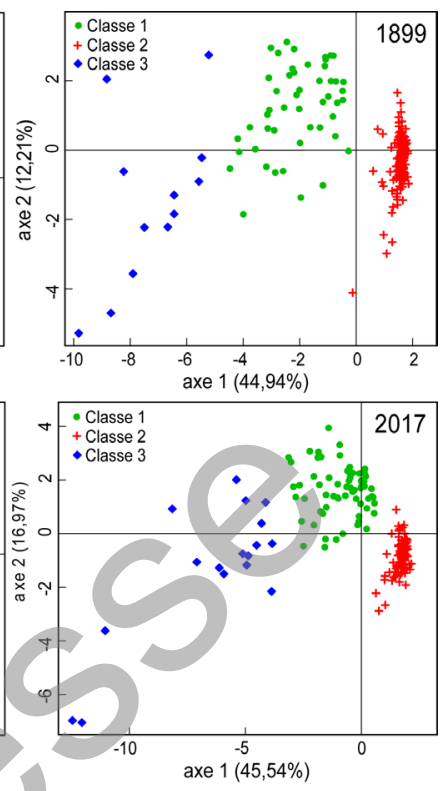

Fig. 8 - Projection des individus sur les deux premiers axes factoriels pour les quatre dates d'analyse $(1866,1899,1972$ et 2017).

Fig. 8 - Projection of individuals on the first two factorial axes (1866, 1899, 1972 and 2017).

4.2. Résultats de la segmentation de la plaine alluviale de la Charente en unités homogènes

Quatre segmentations de la plaine alluviale en unités homogènes ont été produites, une pour chacune des dates (fig. 9). Les dates les plus récentes (1972 et 2017) présentent un patron (pattern) d'organisation très proche, ce qui atteste d'une faible évolution au cours de cette période. Pour les dates les plus anciennes (1866 et 1899), seule la partie centrale du tracé montre des différences significatives. De ce fait, la segmentation en zones homogènes peut être considérée comme stable dans le temps. Elle s'appuie sur l'analyse des quatre segmentations générées et sur une base qualitative pour ajuster les

Fig. 9 - Segmentation de la plaine alluviale de la Charente par détection automatique de ruptures spatiales.

Fig. 9 - Segmentation of the alluvial plain of the Charente River by automatic detection of spatial rupture. 
limites des zones. Le linéaire a par conséquent été découpé en quatre zones :

- la zone 1 (Z1) entre Angoulême et Châteauneuf-sur-Charente (63 tronçons)

- la zone 2 (Z2) entre Angeac-Charente et Bourg-Charente (33 tronçons)

- $\quad$ la zone 3 (Z3) entre Saint-Brice et Merpins (28 tronçons)

- la zone 4 (Z4) entre Saint-Laurent-de-Cognac et Saintes (56 tronçons)
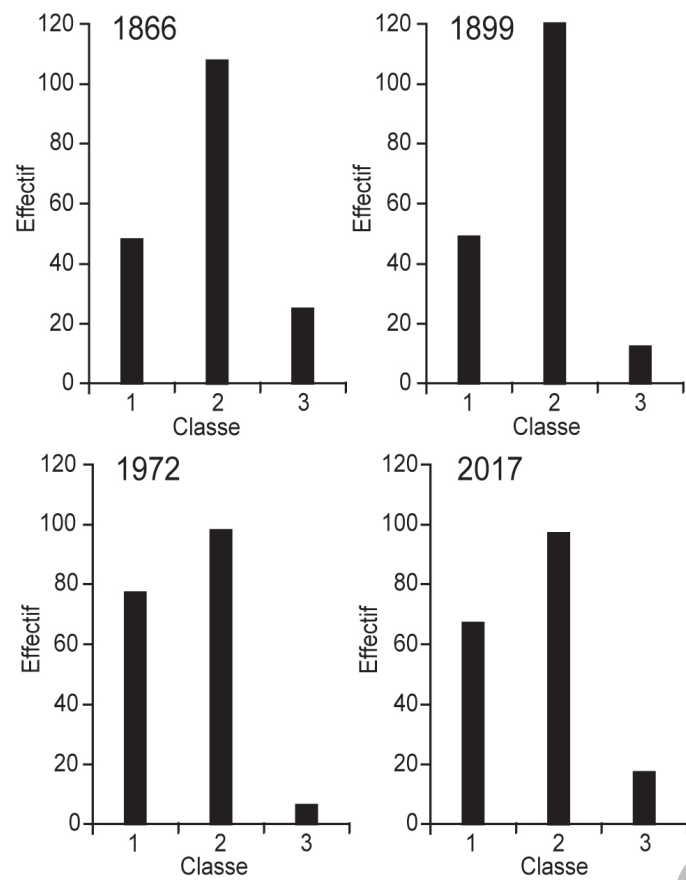

Fig. 10 - Répartition des classes de la typologie à léchelle du linéaire de la Charente.

Fig. 10 - Distribution of the typology classes at the Charente River scale.

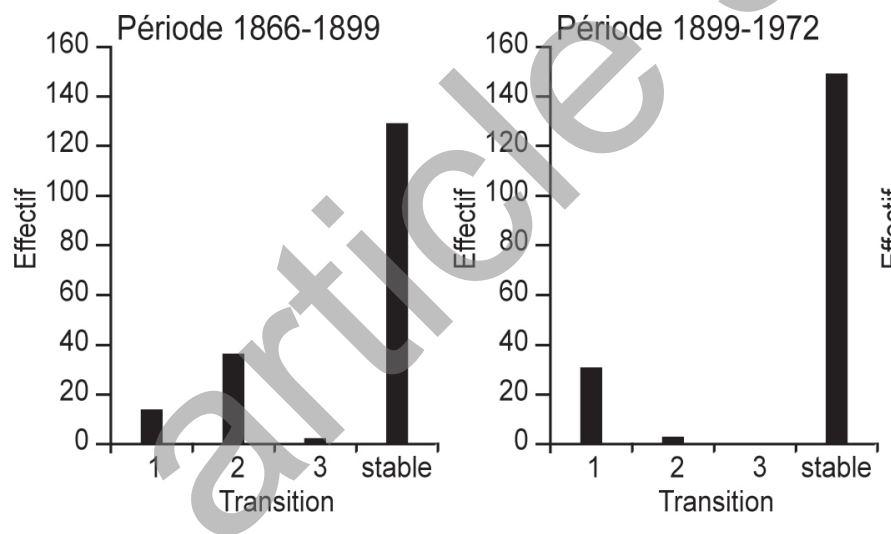

4.4. Analyse spatiale des résultats à l'échelle des zones homogènes

Le linéaire de la Charente a été découpé en 4 zones homogènes. Chacune d'entre elles est caractérisée par la présence ou l'absence d'une ou plusieurs classes de la typologie issue des résultats de l'ACP et de la CAH (fig. 12). Une cartographie de la typologie a également été produite (fig. 13).

La zone Z1 entre Angoulême et Châteauneuf-sur-Charente
4.3. Analyse des résultats à l'échelle du linéaire de la Charente

La répartition des classes montre une prédominance des tronçons fluviaux de classe 2 sur la période 1866-2017 (fig. 10). Ils connaissent une légère augmentation entre 1866 et 1899 (108 à 120 tronçons), une nette diminution entre 1899 et 1972 (120 à 98), et une relative stabilité entre 1972 et 2017 (98 à 97). L'augmentation des tronçons fluviaux de classe 2 en 1899 s'accompagne d'une diminution de moitié des tronçons de classe 3 (12) et d'une stabilisation des tronçons de classe 1 (49). Entre 1899 et 1972, les tronçons fluviaux de classe 1 subissent une forte progression (77) alors que ceux de classe 3 sont peu représentés sur l'ensemble du linéaire (6). Ces derniers connaissent toutefois une légère augmentation (17) en 2017.

Quatre types d'évolution significatifs ou trajectoires s'observent à l'échelle de la Charente (fig. 11). La première montre une augmentation progressive du nombre de tronçons fluviaux stables, de 129 en 1866 à 160 en 2017, ce qui souligne la tendance globale des formes fluviales de la Charente à se stabiliser sur la période d'étude. La deuxième rend compte du passage de 36 tronçons de classe 1 ou 3 vers la classe 2 entre 1866 et 1899 . Ce changement témoigne d'une simplification de la mosaïque fluviale dans la partie amont de la Charente suite à la disparition d'un certain nombre d'îles et de chenaux secondaires. La troisième évolution révèle la transformation de 30 tronçons (dont 24 de classe 2) vers la classe 1 entre 1899 et 1972 liée à la formation d'îles nouvelles. Enfin, la dernière montre l'évolution de tronçons à chenal unique (7) vers des tronçons anastomosés de la classe 3 entre 1972 et 2017 en raison du démantèlement de francs-bords.

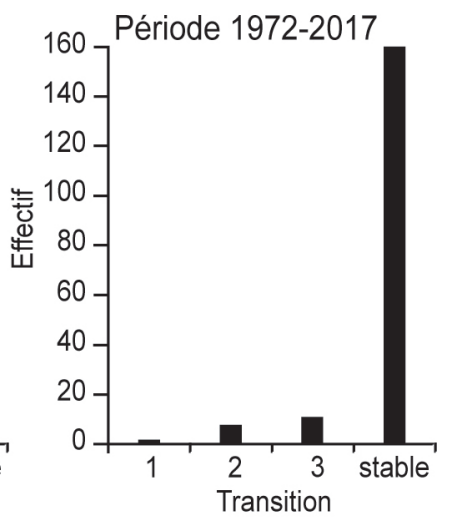

Fig. 11 - Evolution inter-période des transitions des classes de la typologie à l'échelle du linéaire de la Charente.

Fig. 11 - Period change of the typology classes at the Charente River scale.

comprend 63 tronçons (T001-T063). Sur les quatre dates, elle est dominée par la présence des classes 1 et 2 . La classe 3 est représentée de manière marginale et seulement pour les dates les plus anciennes. L'association des classes 1 et 2 sur la zone $\mathrm{Z} 1$ témoigne de la présence de tronçons fluviaux anastomosés à chenaux multiples et étroits avec la présence de nombreux micro-îlots et îlots végétalisés et de tronçons fluviaux à chenal unique méandriforme sur l'ensemble de la période d'étude (fig. 13).

La zone Z2 entre Angeac-Charente et Bourg-Charente comprend 

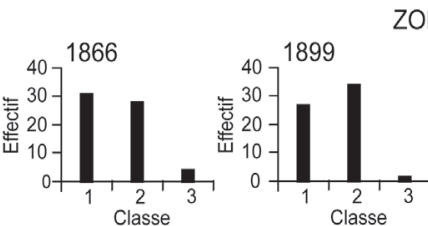

ZONE 1
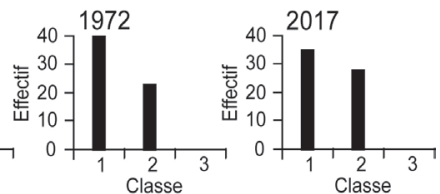

ZONE 2
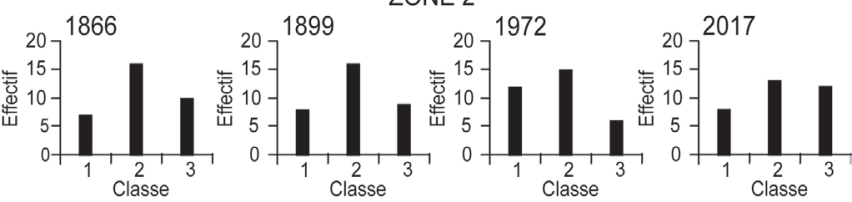

ZONE 3
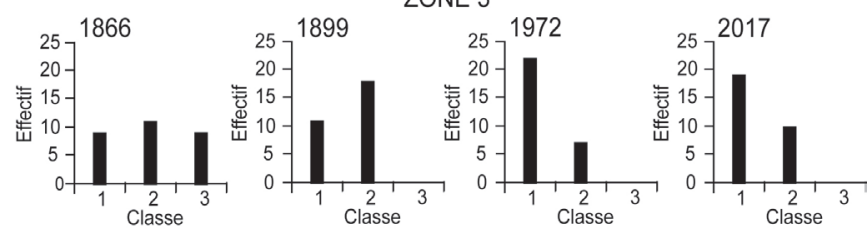

ZONE 4
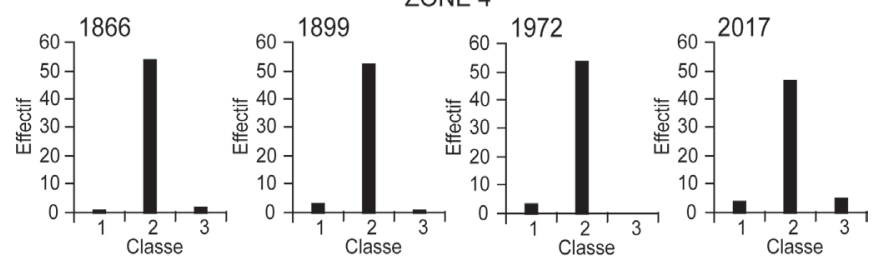

LINEAIRE TOTAL
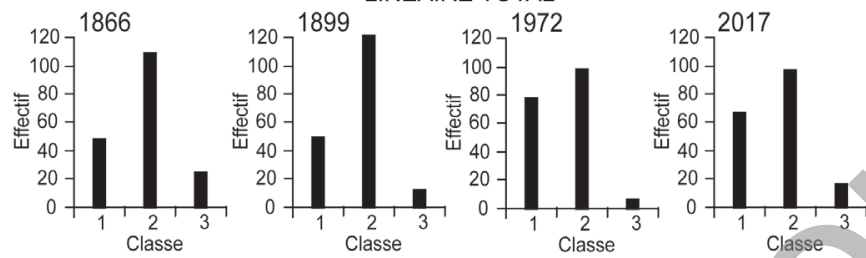

Fig. 12 - Répartition des classes de la typologie à l'échelle des zones homogènes et du linéaire de la Charente.

Fig. 12 - Distribution of typology classes at the homogeneous units and Charente River scale.

33 tronçons (T064-T096). Elle se distingue par la prédominance de la classe 3 aux 4 dates (10 en 1866, 9 en 1899, 6 en 1972 et 12 en 2017) (fig. 12). Elle est caractérisée par des tronçons anastomosés marqués par le développement d'îles végétalisées de grande dimension sur la période 1866-2017.

La zone Z3 entre Saint-Brice et Merpins est composée de 28 tronçons (T097-T125). Elle se distingue principalement par l'association des classes 1 et 2. La zone Z3 présente des tronçons à chenal unique et des tronçons anastomosés avec des îles végétalisées de taille moyenne développées sur une bande fluviale et une plaine alluviale réduites. Certains tronçons anastomosés de cette zone font partie de la classe 1 pour les années 1899, 1972 et 2017 mais certains d'entre eux migrent vers la classe 3 en 1866 (fig. 13). Cette trajectoire s'expliquerait par un biais lié à la méthode : e.g., erreur brute des données et erreur de digitalisation.

La zone Z4 entre Saint-Laurent-de-Cognac et Saintes inclut 56 tronçons (T126-T181). Elle est marquée par la prédominance de la classe 2 sur l'ensemble du linéaire aval pour les 4 années (53 en 1866, 52 en 1899, 53 en 1972 et 47 en 2017) (fig. 12). Seuls 2 secteurs s'individualisent par leur appartenance aux classes 1 et 3 : les 3 tronçons T155-T157 sur la période 1866-2017 et les 6 tronçons T169-T174 en 2017 (fig. 13).

\subsection{Trajectoire(s) d'évolution de la Charente de 1866 à nos jours}

81 modèles de trajectoire d'évolution théoriques sont possibles à partir de la typologie en 3 classes pour les 4 dates. Dans la réalité, seules 22 trajectoires d'évolution sont observées sur l'ensemble de la Charente (tab. 2). Les trajectoires 1, 2 et 3 sont prédominantes, ce qui révèle la stabilité du chenal et des îles à l'échelle du linéaire sur la période 1866-2017. 120 tronçons fluviaux (66,3\%) conservent leur classe d'origine : 31 tronçons pour la classe 1, 84 pour la classe 2 et 5 pour la classe 3. 61 tronçons enregistrent par conséquent un ou plusieurs changements de classe dans leur trajectoire. Les modèles 4, 5, 6, 7 et 8 sont également représentés et comprennent respectivement 12, 9, 6, 5 et 7 tronçons. Les 14 autres modèles de trajectoire d'évolution observés sont quant à eux marginaux. Ils concernent seulement 1 à 3 tronçons au sein du linéaire fluvial.

\section{Interprétation}

L'analyse quantitative cartographique permet de restituer la trajectoire d'évolution à l'échelle du linéaire de la Charente entre Angoulême et Saintes et de mettre en évidence quatre trajectoires d'évolution distinctes au sein de chaque zone homogène sur la période 1866-2017. Malgré les limites liées à la méthode utilisée, les résultats obtenus confirment la stabilité du système fluvial charentais au cours des 150 dernières années. Toutefois l'analyse factorielle des données géomorphométriques par ACP et $\mathrm{CAH}$ souligne l'existence de plusieurs tronçons fluviaux aux modèles de trajectoire d'évolution singuliers liés à un ou plusieurs changements d'appartenance de classe depuis 1866 associés à des processus d'ajustements fluviaux.

La confrontation de ces résultats avec l'histoire des aménagements et des usages du fleuve Charente reconstituée à partir des sources archivistiques (correspondances, notes, rapports, minutes, descriptions de projets, plaintes de riverains), iconographiques (cartes, plans, coupes, élévations, cartes postales, photographies aériennes) et bibliographiques permet de proposer une explication aux tendances évolutives observées à l'échelle du linéaire, des zones homogènes et des tronçons fluviaux.

\subsection{La navigation fluviale, principal facteur de forçage an- thropique}

L'analyse qualitative des sources historiques permet de reconstituer une chronologie des aménagements et actions pour rétablir et/ou garantir la navigation de la Charente présentée dans la Figure 14. Elles sont ensuite mises en relation avec les processus d'ajustement fluvial observés à l'échelle du linéaire, des zones homogènes et des tronçons fluviaux.

\subsubsection{Période 1866-1899}

Un phénomène de simplification et d'homogénéisation de la mosaïque fluviale est observé à l'échelle du linéaire de la Charente entre 1866 et 1899 . Cette observation semble cohérente avec les interventions des sociétés pour la navigation, décrites dans les sources d'archives. Elles sont prédominantes sur la période 1838- 


\section{ZONE Z4}

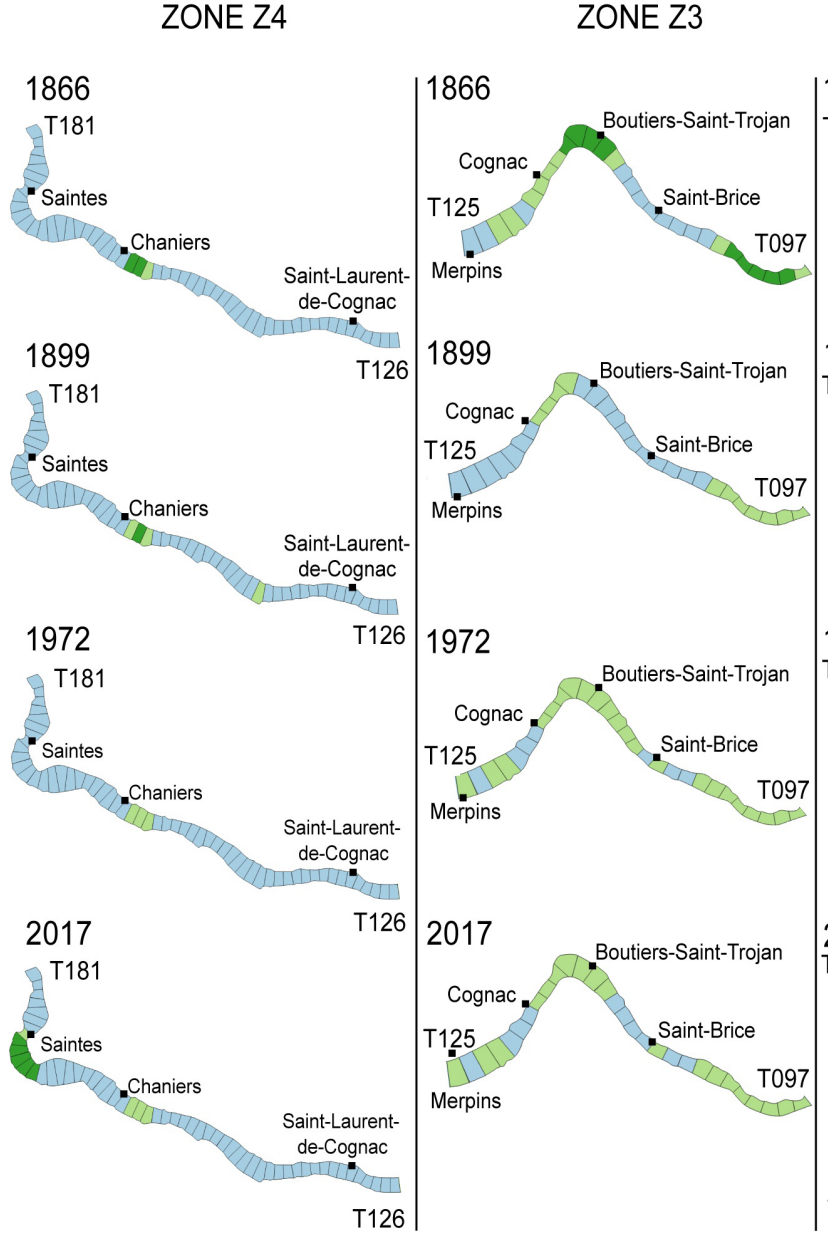

ZONE Z2

ZONE Z1

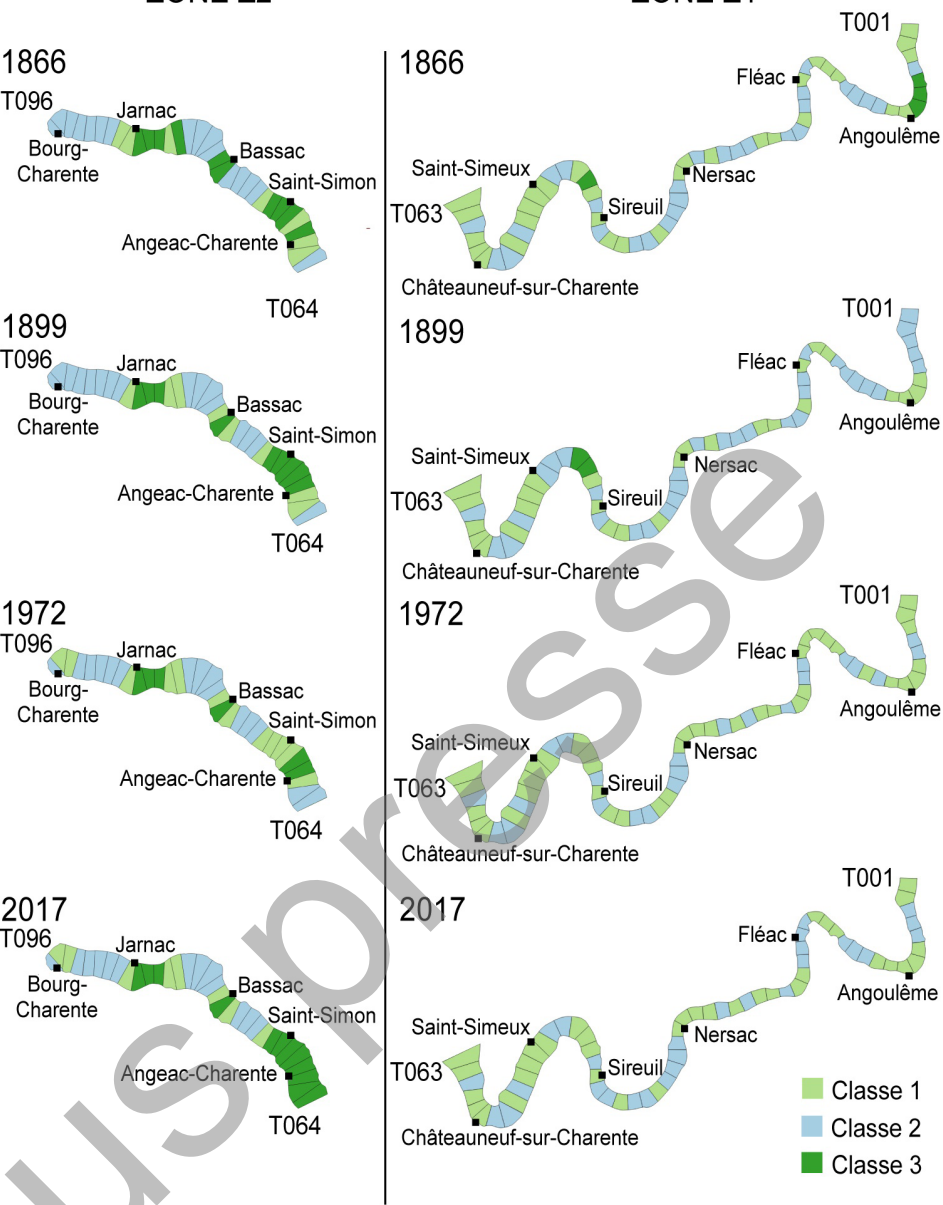

Fig. 13 - Spatialisation de la typologie à l'échelle des zones Z1, Z2, Z3 et Z4 entre 1866 et 2017.

Fig. 13 - Spatialization of typology at the scale of units Z1, Z2, Z3 and Z4 between 1866 and 2017.

1859 consécutivement à la loi d'amélioration de la Charente pour la navigation (24 mars 1825) pour permettre l'exportation des eauxde-vie charentaises (cognac) et de la papeterie angoumoisine (papier à cigarette et production haut de gamme pour les enveloppes) et sur la période 1881-1891 suite au décret du 17 mars 1881 sur le linéaire Cognac-Saintes (fig. 14). Ces interventions consistent à maintenir le chenal, les berges et les chemins de halage et à garantir le libre écoulement des eaux (dragages, réaménagements portuaires, entretien de la végétation des berges et des chemins de halage, aménagement d'ouvrages en travers, etc.). Ainsi, la simplification et l'homogénéisation du système fluvial se traduisent par trois processus d'ajustement majeurs :

(i) la stabilité du chenal s'observe sur les 4 zones du linéaire, mais elle est prédominante dans les zones $\mathrm{Z} 2$ et $\mathrm{Z} 4$ où la plupart des tronçons fluviaux conserve une trajectoire d'évolution stable.

(ii) la destruction des unités fluviales les plus fragiles se produit dans les zones $\mathrm{Z} 1$ et $\mathrm{Z} 3$ et dans une moindre mesure, dans la zone Z2. Les tronçons T001, T002 et T003 en amont d'Angoulême (zone Z1), T089 à Jarnac (zone Z2), et T120 et T119 en aval de Cognac (zone Z3) évoluent d'un style à anastomose simple en 1866 vers un style à chenal unique méandriforme en 1899. La disparition des bancs non végétalisés et des îlots enherbés peut être attribuée aux actions volontaires de suppression des obstacles naturels à la navigation soutenues par les Ponts et Chaussées car leur présence diminue la largeur des chenaux et affaiblit le tirant d'eau contraignant le passage des gabarres. Leur disparition qui induit un élargissement ponctuel du chenal en eau principal, agit également sur le transfert de la charge solide à l'échelle du linéaire et du bassin versant à cette période car les pertes en sédiments par prélèvements ne sont pas compensées par les apports sédimentaires.

(iii) le rattachement à la berge des îles n'est identifié que dans la zone Z2 au sein des tronçons T120-T119 en aval de Cognac; aucune information ne permet de dire s'il est d'origine anthropique (fermeture du bras secondaire à des fins de navigation ou/et à des fins agricoles pour étendre la superficie cultivable) ou d'origine naturelle (déconnexion du bras secondaire et végétalisation en l'absence d'entretien).

\subsubsection{Période $1899-1972$}

La période 1899-1972 est caractérisée par la poursuite de la simplification et d'homogénéisation du paysage fluvial et par un phénomène de complexification de la mosaïque fluviale à l'échelle du linéaire de la Charente. Ces phénomènes montrent un système fluvial laissé peu à peu à l'abandon après l'arrêt progressif de la navigation et des pratiques d'entretien du chenal, des berges et des chemins de halage amorcé à la fin du XIX ${ }^{e}$ siècle. La navigation fluviale ne parvient pas à concurrencer le transport ferroviaire en 
Tab. 2 - Modèles de trajectoire d'évolution des tronçons de la Charente sur la période 1866-2017.

Tab. 2 - Evolutionary trajectory models 'units of the Charente River during the period 1866-2017.

\begin{tabular}{|c|c|c|c|c|c|}
\hline \multicolumn{5}{|c|}{$\begin{array}{l}\text { Modèles de trajectoire } \\
\text { d'évolution stables }\end{array}$} & \multirow[b]{2}{*}{$\begin{array}{c}\text { Nombre de } \\
\text { tronçons fluviaux }\end{array}$} \\
\hline $\begin{array}{l}\text { Modèle de } \\
\text { transition }\end{array}$ & 1866 & 1899 & 1972 & 2017 & \\
\hline 1 & 1 & 1 & 1 & 1 & 31 \\
\hline 2 & 2 & 2 & 2 & 2 & 84 \\
\hline 3 & 3 & 3 & 3 & 3 & 5 \\
\hline \multicolumn{6}{|c|}{$\begin{array}{l}\text { Modèles de trajectoire } \\
\text { d'évolution courants }\end{array}$} \\
\hline $\begin{array}{l}\text { Modèle de } \\
\text { transition }\end{array}$ & 1866 & 1899 & 1972 & 2017 & $\begin{array}{c}\text { Nombre de } \\
\text { tronçons fluviaux }\end{array}$ \\
\hline 4 & 3 & 1 & 1 & 1 & 12 \\
\hline 5 & 2 & 2 & 1 & 1 & 9 \\
\hline 6 & 2 & 2 & 2 & 3 & 6 \\
\hline 7 & 2 & 2 & 1 & 2 & 5 \\
\hline 8 & 1 & 2 & 1 & 1 & 7 \\
\hline \multicolumn{6}{|c|}{$\begin{array}{l}\text { Modèles de trajectoire } \\
\text { d'évolution marginaux }\end{array}$} \\
\hline $\begin{array}{l}\text { Modèle de } \\
\text { transition }\end{array}$ & 1866 & 1899 & 1972 & 2017 & $\begin{array}{c}\text { Nombre de } \\
\text { tronçons fluviaux }\end{array}$ \\
\hline 9 & 1 & 2 & 1 & 2 & 1 \\
\hline 10 & 3 & 2 & 2 & 2 & 1 \\
\hline 11 & 2 & 2 & 2 & 1 & 2 \\
\hline 12 & 1 & 1 & 1 & 2 & 2 \\
\hline 13 & 3 & 3 & 1 & 1 & 2 \\
\hline 14 & 1 & 3 & 1 & 1 & 1 \\
\hline 15 & 1 & 1 & 2 & 3 & 1 \\
\hline 16 & 1 & 1 & 1 & 2 & 1 \\
\hline 17 & 1 & 3 & & & 1 \\
\hline 18 & 3 & 3 & & 3 & 3 \\
\hline 19 & 2 & & 1 & 1 & 1 \\
\hline 20 & 1 & & & 2 & 3 \\
\hline 21 & & (2) & 1 & 1 & 2 \\
\hline 22 & & & 2 & 2 & 1 \\
\hline
\end{tabular}

pleine expansion depuis l'ouverture de la ligne de chemin de fer Angoulême-Rochefort-La Rochelle par la compagnie des Deux Charentes en 1867. La Charente est alors déclassée en amont de Cognac en 1926 et la totalité du cours d'eau en 1957 (fig. 14).

La simplification et l'homogénéisation des formes fluviales se manifestent par:

(i) une stabilisation des formes fluviales sur les 4 zones, mais elle reste prédominante dans les zones $\mathrm{Z1}, \mathrm{Z} 2$ et $\mathrm{Z} 4$.

(ii) une végétalisation des berges et des îles par les formations arborées qui apparaît en premier dans les zones les plus en amont du fleuve et particulièrement au sein des tronçons marqués par une stabilisation du chenal et des îles dans les zones Z1 et Z2. Elle s'est amorcée après l'abandon des pratiques d'entretien des berges et des chemins de halage au moment du déclin du trafic fluvial. Mais, l'enforestement des îles et des berges reste modéré et contenu par les pratiques agro-pastorales.

(iii) un rattachement à la berge des îles : il concerne uniquement la zone Z2 sur les tronçons T069, T070 et T071. Ces tronçons passent d'une anastomose complexe stable sur la période 1866-1899 à un style à anastomose simple en 1972 marqué par la suppression d'un chenal secondaire après une intervention anthropique pour la navigation.

La complexification de la mosaïque fluviale concerne la partie supérieure de la Charente (zones Z1, Z2 et Z3). Elle se traduit par un processus d'apparition de nouvelles unités fluviales et par un processus de détachement d'îles de la berge. L'apparition d'unités fluviales concerne la formation de nouvelles unités fluviales enherbées de type micro-îlots et îlots non issus d'une division ou d'un détachement de la berge et formées par des dépôts d'alluvions. Seule la zone la plus en amont (zone Z1) et la zone médiane (zone Z3) du cours d'eau sont concernées; jusqu'au début du XX $\mathrm{XX}^{\mathrm{e}}$ siècle la partie en aval de Cognac (zone Z4) est restée entretenue pour la navigation (transport des eaux-de-vie du port de Cognac vers Rochefort). Ces modifications sont attribuées, à défaut d'autres renseignements, à un envasement du lit mineur (Jouannet, 1992) dans les zones à faible énergie colonisées progressivement par une végétation spontanée herbacée et arbustive au cours de la période 1899-1972. Le processus de détachement des îles de la berge se rencontre dans la zone Z2. Les tronçons T094-T095 observent une transformation de leur trajectoire d'évolution : passage d'un style à chenal unique méandriforme à un style à anastomose simple à la suite de l'apparition d'une île boisée large de forme relativement circulaire. Faute d'archives, ce détachement est attribué à une intervention humaine en raison de la rectitude du chenal secondaire.

\subsubsection{Période 1972-2017}

La période 1972-2017 est également caractérisée par un phénomène de simplification des formes fluviales à l'échelle du linéaire. Ce dernier pourrait être attribué au reclassement de la Charente pour la navigation de plaisance en 1970 à la demande des départements de Charente et Charente-Maritime et de la Région Poitou-Charentes. La relative stabilité de la bande fluviale, l'élargissement du chenal en eau principal et la stabilité ou la réduction des surfaces en île seraient le résultat du retour des pratiques d'entretien régulières du chenal et des îles (curages, entretien des berges et des chemins de halage, élagage, recépage et plantation d'arbres, etc.) afin de garantir le libre écoulement des eaux. La stabilité des formes fluviales s'observe sur les quatre zones sans véritable distinction étant donné que 160 sur 181 tronçons ont conservé leur style fluvial par rapport à 1972. La disparition des unités fluviales les plus fragiles a lieu uniquement dans les zones Z1 (T003, T011, T017, T018, T036 et T041) et Z3 (T109, T110 et T111). Le style à anastomose simple est remplacé par un style à chenal unique méandriforme. Cette transformation pourrait être attribuée à la reprise des pratiques d'entretien du chenal (disparition des micro-îlots et des îlots enherbés apparus en 1972). Le processus de détachement des îles de la berge s'observe dans la zone Z2 (T064, T065 et T066). Une grande île végétalisée 


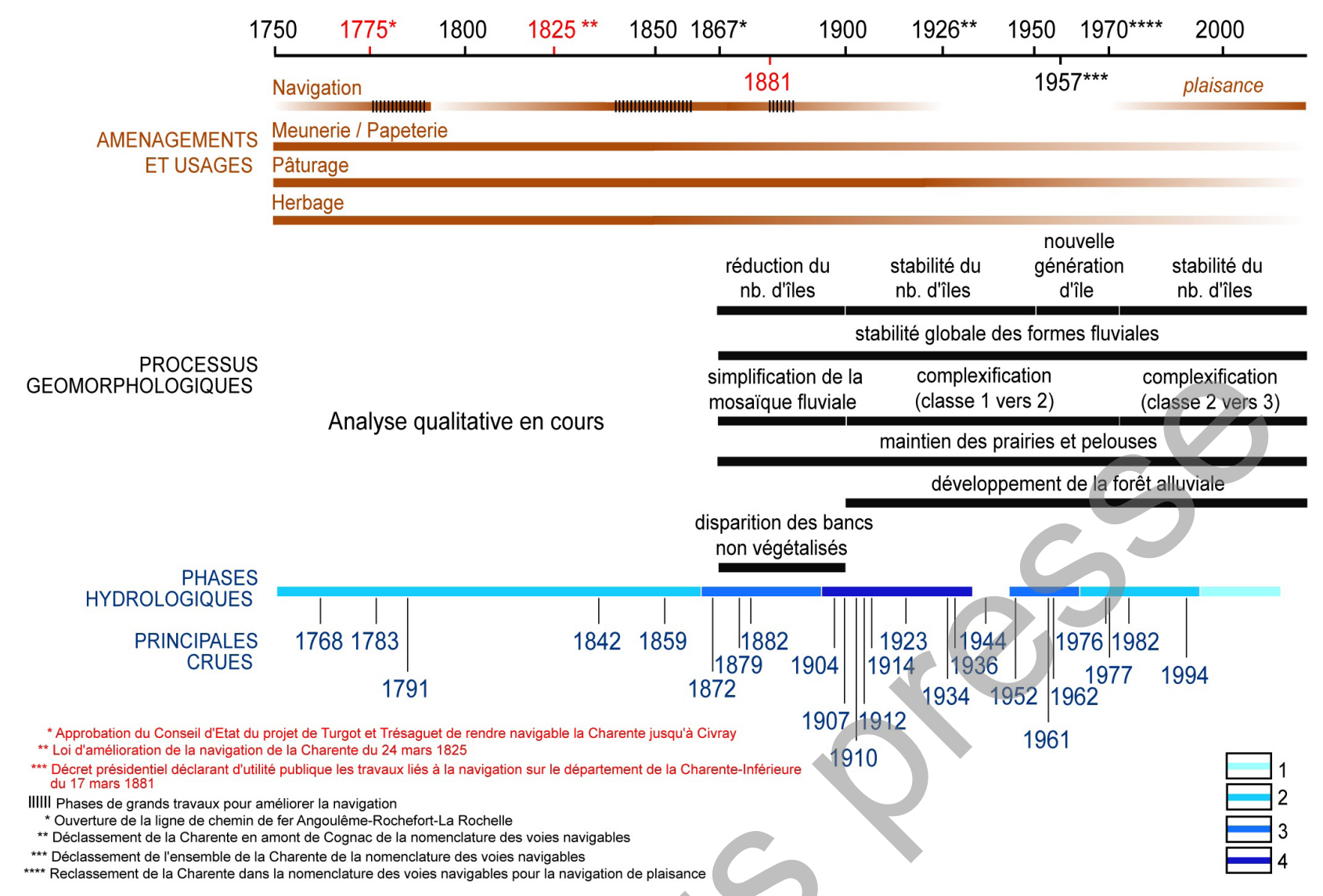

Fig. 14 - Evolution morphologique de la Charente en liaison avec les forçages anthropiques et climatiques.

1. Période d'activité hydrologique réduite. 2. Période de moindre activité hydrologique ponctuée de crues extrêmes. 3. Période de forte activité hydrologique marquée par des crues courantes. 4. Période de forte activité hydrologique marquée par des crues courantes et extrêmes.

Fig. 14 - Morphological evolution of the Charente River related to anthropogenic and climatic factors.

1. Period of low hydrological activity. 2. Period of average hydrological activity characterized by extreme floods. 3. Period of strong hydrological activity characterized by common floods. 4. Period of strong hydrological characterized by common and extreme floods.

pour laquelle il s'était opéré un processus de rattachement à la berge au cours du XIX ${ }^{\mathrm{e}}$ siècle suite au comblement du bras secondaire, s'est détachée de la berge. Il pourrait être lié à une reprise de l'entretien des chenaux secondaires jusque-là laissés à l'abandon et colonisés par une végétation continue. La végétalisation des îles et des berges s'est renforcée sur les zones Z1, Z2 et Z3. Elle laisse envisager une stabilité du système fluvial et souligne une dynamique volontaire de fermeture des milieux par la plantation de peupliers à des fins de mise en valeur forestière, paysagère et touristique.

La reprise de la navigation sur la Charente s'est produite sur une période récente. Certes, certains effets sont déjà observables sur ce système fluvial à très faible énergie (e.g., disparition des unités fluviales, détachement des îles de la berge, etc.) mais d'autres ne le sont peut-être pas encore, par exemple, la déstabilisation des berges.

\subsection{Autres facteurs de forçage anthropiques}

\subsubsection{Moulinage et usages agro-pastoraux}

La part du moulinage et des usages agro-pastoraux reste difficile à qualifier et à quantifier au sein du linéaire fluvial charentais en raison de la rareté des sources géohistoriques. La navigation est en effet la principale cause des actions sur la Charente et elle est par conséquent une grande pourvoyeuse d'archives. Ainsi, fautes de données et d'études antérieures, ces deux facteurs de forçage anthropique ne sont donc pas abordés dans le cadre de ce travail. Toutefois des travaux menés à l'échelle de deux sites d'étude (Île de la Baine à Chaniers et Île Domange à Angéac-Charente) permettront de mieux comprendre l'impact des moulins et de leurs ouvrages (seuils, déversoirs, etc.) sur un système fluvial à faible énergie et à anastomose. L'acquisition de données de terrain (carottages, TRE, EM31, bathymétrie monofaisceaux) permettra de pallier l'absence de données géohistoriques.

\subsubsection{Protection contre les inondations}

Pour la période 1866-1972, la faiblesse des sources écrites ne permet pas de mesurer l'impact des aménagements de lutte contre les inondations. Bien que la Charente fasse l'objet d'inondations fréquentes aux conséquences parfois désastreuses (fig. 14), plusieurs projets sont présentés par le service des Ponts-et-Chaussées, mais aucun n'est suivi par des mesures effectives. Cette inactivité est due aux spécificités du régime de crues (débits peu élevés, montée de crue lente, vitesses d'écoulement réduites), à une politique d'aménagement tournée essentiellement vers la navigation jusqu'à son abandon, à la complexité des projets envisagés, à leur coût 
important et enfin, à une perception positive des crues car elles conduisent à un enrichissement naturel des terres agricoles en limons. Seules des mesures ponctuelles et à coût réduit (pieux et palplanches) sont par conséquent mises en œuvre par les riverains pour fixer les berges érodées par les courants en temps de crue en particulier dans la partie amont du cours d'eau (zone Z2) sur la période 1866-1899.

Pour la période 1972-2017, c'est à partir de la crue de 1982 que les aménagements de protection contre les inondations deviennent un facteur d'évolution de la trajectoire de certains tronçons fluviaux. C'est le cas pour les tronçons T169-T174 au niveau de Saintes situés dans la zone Z4. Ils observent le modèle de transition $n^{\circ} 6$ pour les tronçons T169-173 (tab. 3) : un style à chenal unique méandriforme stable sur la période 1866-1972 évoluant vers un style à anastomose entre 1972 et 2017 suite à l'apparition d'une île artificielle végétalisée de grande dimension (1 $\left.159320 \mathrm{~m}^{2}\right)$ et de forme allongée (6525 $\mathrm{m})$. Cette île est issue du détachement d'un franc-bord suite à l'aménagement d'un canal de dérivation en 1986 pour améliorer l'écoulement des eaux en temps de crue et de protéger la ville de Saintes contre les inondations de type 1982 (hauteur de 6,84 m à l'échelle amont du pont Palissy). Le tronçon T174 observe quant à lui le modèle $n^{\circ} 11$ : il passe d'un style à chenal unique méandriforme stable sur la période 1866-1972 à un style à anastomose entre 1972 et 2017 avec la présence d'une île enherbée dont l'origine serait attribuée au détachement de l'extrémité aval de l'île par érosion.

\section{Conclusion}

Cette étude présente une première analyse de l'évolution de la moyenne Charente de 1866 jusqu'à nos jours. Les résultats obtenus montrent une stabilité des chenaux et des îles sur les deux derniers siècles. Cette dernière peut être interprétée comme la conjonction entre : (i) la très faible énergie du système fluvial qui ne dépasse pas $10 \mathrm{~W} \cdot \mathrm{m}^{-2}$, cette faible énergie est liée à la conjonction d’une pente faible et d'un débit à plein-bord modeste; et (ii) la cohésion des berges qui sont principalement limoneuses et stabilisées par la végétation. Dans ce contexte, la stabilité dû système serait essentiellement rompue par les interventions anthropiques. A l'échelle du linéaire, les évolutions observées semblent être commandées en majeure partie par la navigation (écluses, quais, cales, canaux de dérivation) et les pratiques d'entretien du lit et des îles qui y sont associées (dragages, suppression des atterrissements, coupes et élagages, etc.). La part des autres facteurs de contrôle anthropique - usages agro-pastoraux, moulinage et protections contre les inondations - est plus difficile à qualifier et à quantifier au sein d'un système fluvial où la navigation est le moteur principal des interventions et par conséquent la principale source d'archives. Les travaux menés à l'échelle de sites (île de la Baine à Chaniers et Île Domange à AngéacCharente) permettront de mieux appréhender ces phénomènes.

Ces résultats sur la moyenne Charente mettent en évidence la nécessité d'adapter une approche géomorphométrique sur des cours d'eau à très faible énergie jusque-là mise en œuvre sur des systèmes fluviaux à moyenne ou forte énergie, sans pour autant remettre en cause sa pertinence. Toutefois, les limites intrinsèques de la méthode doivent être prises en compte dans l'interprétation des résultats obtenus. Ainsi, l'existence d'éventuels ajustements du système fluvial est parfois difficile à observer car ils sont proches ou inférieurs à l'incertitude générée par la méthode. La méthode statistique ne permet pas d'identifier les facteurs de contrôle mais seulement de proposer des pistes de réflexion pour expliquer les tendances évolutives observées et nécessite la mise en œuvre d'une enquête géohistorique en complément. Enfin, l'analyse du rôle des crues sur l'évolution de la moyenne Charente reste à explorer afin d'évaluer leur rôle dans cette dynamique fluviale réduite. Les chroniques de crues reconstituées sur la période 1700-2018 (Duquesne et Carozza, 2019), permettront d'analyser leur impact. Malgré ces limites, l'approche quantitative proposée démontre dans tous les cas l'extrême faiblesse des ajustements morphologiques du chenal (ce travail) et des îles de la Charente.

Enfin, ces travaux sur une durée de deux siècles devront être complétés par une analyse qualitative sur la période 1600-2017 à partir des cartes les plus anciennes afin de démontrer que les tendances observées aux $\mathrm{XIX}^{e}$ et $\mathrm{XX}^{e}$ siècles s'inscrivent sur une échelle de temps longue. Le recours à l'utilisation seule de cartes anciennes et de sources textuelles pour expliquer les ruptures d'évolutions observées reste toutefois dépendant de la disponibilité et de l'accessibilité des documents, de l'imprécision des cartes anciennes, de l'absence d'informations pour contextualiser les cartes et de leur subjectivité.

Ces premiers résultats montrent que la Charente moyenne est bien une rivière paradoxale. Le maintien jusqu’à la période actuelle de son style à anastomose est une spécificité propre à ce fleuve. A rebours de Lewin (2010) et Brown et al. (2018) qui indiquent que les paysages fluviaux à anastomose sont des reliques de paysages fluviaux peu modifiées par l'intervention humaine et dont le maintien serait expliqué par des conditions géologiques et/ou d'isolement particulières, la Charente montre que les anastomoses ont pu, au moins partiellement, perdurer dans un contexte de forte anthropisation. Loin de constituer une marge, le fleuve Charente, "le plus beau fleuve de France " d'après François I ${ }^{\mathrm{er}}$, a fait l'objet d'aménagements et d'un entretien massif du chenal, des berges et de la plaine alluviale pour la navigation, le transport par voie terrestre, l'exploitation de la force motrice de l'eau (moulins et papeteries) et la subsistance des populations (pêcheries) sur plusieurs millénaires (Dumont et Mariotti, 2013). Cependant, il reste encore difficile de quantifier l'impact de la simplification de la mosaïque fluviale notamment au cours du dernier millénaire et d'envisager son importance à la fois dans l'espace et dans le temps. L'adaptation des aménagements des usages et des pratiques aux spécificités de l'écoulement et de la morphologie du cours d'eau et donc, la co-innovation, pourrait être une explication à cette apparente contradiction.

\section{Références}

Albert A., Piégay H. (2011) - Spatial disaggregation and aggregation procedures for characterizing fluvial features at the networkscale: Application to the Rhône basin (France). Geomorphology, 125 (3), 343-360.

DOI : 10.1016/j.geomorph.2010.09.009

Aucelli P.P.C., Fortini P., Rosskopf C.M., Scorpio V., Viscosi V. (2011) - Recent channel adjustments and riparian vegetation: Some examples from Molise (Italy). Geografia Fisica e Dinamica Quaternaria, 34 (2), 161-173. 
DOI : $10.4461 /$ GFDQ.2011.34.15

Beauchamp A., Lespez L., Rollet A. J., Germain-Vallée C., Delahaye D. (2017) - Les transformations anthropiques d'un cours d'eau à faible énergie et leurs conséquences, approche géomorphologique et géoarchéologique dans la moyenne vallée de la Seulles, Normandie. Géomorphologie : relief, processus, environnement, 23 (2), 1-30.

DOI : $10.4000 /$ geomorphologie.11702

Billaud Y. (2010) - Le Néolithique ancien du site subaquatique de Saint-Simon : le site de l'île des Bois, Charente, L'Archéo thema revue d'archéologie et d'histoire, 6, 6-7.

Bray D. J. (1987) - A study of channel changes in a reach of the North Nashwaaksis stream, New Brunswick, Canada. Earth Surface Processes and Landforms, 12 (2), 151-165.

DOI : 10.1002/esp.3290120205

Brown A. G., Lespez L., Sear D. A., Macaire J. J., Houben P., Klimek K., Brazier R. E., Van Oost K., Pears B. (2018) Natural vs Anthropogenic Streams in Europe: History, Ecology and Implications for Restoration, River-Rewilding and Riverine Ecosystem Services. Earth-Science Reviews, 180, 185-205.

DOI : 10.1016/j.earscirev.2018.02.001

Brunier G., Anthony E. J., Goichot M., Provansal M., Dussouillez P. (2014) - Recent morphological changes in the Mekong and Bassac river channels, Mekong Delta: The marked impact of river-bed mining and implications for delta destabilisation. Geomorphology, 224 (177), 177-191.

DOI : 10.1016/j.geomorph.2014.07.009

Bry C., Hoflack P. (2004) - Le bassin versant de la Charente : une illustration des problèmes posés par la gestion quantitative de l'eau, Courrier de l'Environnement de l'INRA, 52, 81-96.

Candel J.H.J. (2020) - Ahead of the curve - Channel pattern formation of low-energy rivers. $\mathrm{PhD}$ Thesis, Wageningen University, $264 \mathrm{p}$.

DOI : $10.18174 / 506616$

Carling P., Jansen J., Meshkova L. (2014) - Multichannel rivers: their definition and classification. Earth Surface Processes and Landforms, 39 (1), 26-37.

DOI : $10.1002 /$ esp.3419

Casin P., Stachowiak C., Marque F. (2011) - Lanalyse en composantes principales de variables non stationnaires. Mathématiques et sciences humaines, 196, 4, 27-40.

DOI : $10.4000 / \mathrm{msh} .12117$

Cellule Migrateurs Charente Seudre (2018) - Cellule migrateurs Charente Seudre. Rapport des actions 2017. Édit. EPTB Charente / Groupement des fédérations de pêche du Poitou-Charentes / CREAA, 144 p.

Comiti F., Da Canal M., Surian N., Mao L., Lenzi M.A. (2011) Channel adjustments and vegetation cover dynamics in a large gravel bed river over the last 200 years. Geomorphology, 125 (1), 147-159.

DOI: 10.1016/j.geomorph.2010.09.011

Comiti F. (2012) - How natural are Alpine mountain rivers? Evidence from the Italian Alps. Earth Surface Processes and Landforms, 37 (7), 693-707.

DOI : $10.1002 /$ esp. 2267

Corbonnois J., Bonnefond M., Chardon V., Rodrigues S., Jugé P., Cali J., Verdun J., Simonetto E., Tchekpo W., Labergerie
E., Faucheux G. (2016) - Détermination des conditions de la dynamique fluviale d'une rivière aménagée de basse énergie, à partir de secteurs du Loir (Bassin de la Loire aval). Géomorphologie : relief, processus, environnement, 22 (4), 345361.

DOI : 10.1016/j.jhydrol.2018.06.019

Dabrin A. (2009) - Mécanismes de transfert des éléments traces métalliques (ETM) et réactivité estuarienne - Cas des systèmes Gironde, Charente, Seudre et Baie de Marennes Oléron. Thèse de doctorat, Université Bordeaux 3, 387 p.

David M., Labenne A., Carozza J.M., Valette P. (2016) Evolutionary trajectory of channel planforms in the middle Garonne River (Toulouse SW France) over a 130-year period: Contribution of mixed multiple factor analysis (MFAmix). Geomorphology, 258, 21-39.

DOI : 10.1016/j.geomorph.2016.01.012

Deconinck A. (2019) - Récit de prospections en aval de Saintes, Arcades, Hors-Série, 22-25.

Dépret T. (2014) - Fonctionnement morphodynamique actuel et historique des méandres du Cher. Thèse de doctorat, Université de Paris I Panthéon-Sorbonne, 514 p.

Dépret T., Gautier E., Hooke J., Grancher D., Virmoux C. (2015) - Hydrological controls on the morphogenesis of low-energy meanders (Cher River, France). Journal of Hydrology, 531 (3), 887-891.

DOI : 10.1016/j.jhydrol.2015.10.035

Dépret T., Gautier E., Hooke J., Grancher D., Virmoux C., Brunstein D. (2017) - Causes of planform stability of a lowenergy-meandering gravel-bed river (Cher River, France). Geomorphology, 285, 58-81.

DOI : 10.1016/j.geomorph.2017.01.035

Dumont A., Mariotti J. F. (2013) - Archéologie et histoire du fleuve Charente : Taillebourg - Port d'Envaux : une zone portuaire du haut Moyen-Âge sur le fleuve Charente. Editions Universitaires de Dijon, Dijon, 316 p.

Duquesne A., Carozza J. M. (2019) - Ecrire la géohistoire d'un fleuve à faible énergie : les crues de la Charente entre Angoulême et Saintes. Physio-Géo - Géographie Physique et Environnement, $14,57-86$.

DOI : $10.4000 /$ physio-geo.8942

Durand P. (2000) - Approche méthodologique pour l'analyse des littoraux sableux par photo-interprétation. Exemple des plages situées entre l'Aude et l'Hérault (Languedoc, France). PhotoInterprétation, 2000 (1-2), 3-18.

EPTB Charente (2003) - Synthèse de l'étude. Etude des potentialités piscicoles des bassins de la Charente et de la Seudre pour les poissons migrateurs. Etablissement Public Territorial de Bassin Charente, Angoulême, $44 \mathrm{p}$.

EPTB Charente (2004) - Plan de gestion des étiages du bassin de la Charente. Etablissement Public Territorial de Bassin Charente, Angoulême, $58 \mathrm{p}$.

EPTB Charente (2012) - SAGE Charente - Etat initial.Etablissement Public Territorial de Bassin Charente, Angoulême, 47 p.

EPTB Charente (2016) - Stratégie Locale de Gestion du Risque d'Inondation (SLGRI) 2016-2021. Etablissement Public Territorial de Bassin Charente, Angoulême, 123 p.

EPTB Charente (2019) - Rapport de suivi du PGE Charente : 
bilan de l'étiage 2019. Etablissement Public Territorial de Bassin Charente, Angoulême, $67 \mathrm{p}$.

Everitt B. (1993) - Channel responses to declining flow on the Rio Grande between Ft. Quitman and Presidio, Texas. Geomorphology, 6 (3), 225-242.

DOI : 10.1016/0169-555X(93)90048-7

Faye I. B. Nd., Hénaff A., Gourmelon F., Diaw A. T. (2008) Evolution du trait de côte à Nouakchott (Mauritanie) de 1954 à 2005 par photo-interprétation. Norois - Environnement, aménagement, société, 208 (3), 11-27.

DOI : $10.4000 /$ norois. 2146

Faye B., Grelet Y. (1991) - Profils sanitaires en élevage bovin laitier : mise en relation avec une typologie d'exploitation. Etudes et recherche sur les systèmes agraires et le développement, 21, 13-47.

Gaillard F. (2018) - Au fil de la Charente, sur la route du papier. Arcades, Numéro spécial, 64-65.

Gailledreau J.P. (2019) - Les principaux témoignages du Néolithique dans le fleuve Charente. Arcades, Hors-Série, 18-21.

Giret A. (2002) - L'hydrologie de la Charente entre nature et anthropisation (1960-1994). Sécheresse - Science et changements planétaires, 13 (2), 105-112.

Giret A. (2004) - Anthropisation de l'écoulement en PoitouCharentes. Norois - Environnement, aménagement, société, 193 (4), 61-76.

DOI : $10.4000 /$ norois. 745

Golovlyov Pavel, Kornilova E., Krylenko I., Belikov V., Zavadskii A., Fingert E., Borisova N., Morozova E. (2019) - Numerical modeling and forecast of channel changes on the river Lena near city Yakutsk. Proceedings of the International Association of Hydrological Sciences, 381, 65-71.

DOI : 10.5194/piahs-381-65-2019

Grelie J. (1933) - La Charente, étude de fleuve. Annales de Géographie, 42 (235), 44-60.

DOI : 10.3406/geo.1933.10622

Gurnell A. M., Downward S. R. (1994) - Channel planform change on the river Dee meanders, 1876-1992. Regulated rivers: Research \& Management, 9 (4), 187-204.

DOI: $10.1002 / \mathrm{rrr} .3450090402$

Gurnell A. (1997) - Channel Change on the River Dee Meanders, 1946-1992, from the Analysis of Air Photographs. Regulated Rivers Research \& Management, 13 (1), 13-26.

DOI : 10.1002/(SICI)1099-1646(199701)13:1<13::AID-

RRR420>3.0.CO:2-W

Gurnell A., Bussettini M., Camenen B., González del Tánago M., Grabowski R., Hendriks D., Henshaw A., Latapie A., Rinaldi M., Surian N. (2014) - A hierarchical multi-scale framework and indicators of hydromorphological processes and forms. Deliverable 2.1, Part 1, of REFORM (Restoring rivers FOR effective catchment Management), a collaborative project (largescale integrating project) funded by European Commission within the $7^{\text {th }}$ Framework Programme under Grant Agreement 282656, $237 \mathrm{p}$.

DOI : $10.1007 / \mathrm{s} 00027-015-0429-0$

Hadley R. F., Emmett W. W. (1998) - Channel changes downstream from a dam, Journal of the American Water Ressources Association. 34 (3), 629-637.

DOI : 10.1111/j.1752-1688.1998.tb00960.x

Joshi S., Jun X. Y. (2017) - Recent changes in channel morphology of a highly engineered alluvial river - the Lower Mississippi River. Physical Geography, 39 (2), 140-165.

DOI : $10.1080 / 02723646.2017 .1340027$

Jouannet, G. (1992) - Charente, Fleuve et Symbole. Le Croit Vif, Paris, $270 \mathrm{p}$.

Jugie M., Gob F., Virmoux C., Brunstein D., Tamisier V., Le Coeur C., Grancher D. (2018) - Characterizing and quantifying the discontinuous bank erosion of a small low energy river using Structure-from-Motion Photogrammetry and erosion pins. Journal of Hydrology, 563, 418-434.

DOI : 10.1016/j.jhydrol.2018.06.019

Keesstra S. D., Van Huissteden J., Vandenberghe J., Van Dam O., De Gier J., Pleizier I. D. (2005) - Evolution of the morphology of the river Dragonja (SW Slovenia) due to land-use changes. Geomorphology, 69 (1), 191-207.

DOI : 10.1016/j.geomorph.2005.01.004

Kiss T., Blanka V. (2012) - River channel response to climateand human-induced hydrological changes: Case study on the meandering Hernád River, Hungary. Geomorphology, 175-176, 115-125.

DOI : 10.1016/j.geomorph.2012.07.003

Lespez L. (2012) - Paysages et gestion de l'eau : sept millénaires d'histoire des vallées et des plaines littorales en Basse-Normandie. Bibliothèque du Pôle Rural 3, MRSH Caen-Presses Universitaires de Caen, Caen, 333 p.

Lespez L., Viel V., Rollet A. J., Delahaye D. (2015) - The anthropogenic nature of present-day low energy rivers in western France and implications for current restoration projects. Geomorphology, 251, 64-76.

DOI : 10.1016/j.geomorph.2015.05.015

Lespez L., Germaine M. A., Barraud R. (2016) - L'évaluation par les services écosystémiques des rivières ordinaires est-elle durable? VertigO - la revue électronique en sciences de l'environnement, 25, 1-20. DOI : $10.4000 /$ vertigo. 17443

Letuppe J. (2019) - Les données inédites des épaves romaines de Courbiac. Arcades, Hors-Série, 26-29.

Lewin J. (2010) - Medieval environmental impacts and feedbacks: the lowland floodplains of England and Wales. Geoarchaeology, 25 (3), 267-311.

DOI : $10.1002 /$ gea.20308

Liébault F., Piégay H. (2002) - Causes of twentieth century channel narrowing in mountain and piedmont rivers of southeastern France. Earth Surface Processes and Landforms, 27 (4), 425-444. DOI : 10.1002 /esp.328

Letortu P. (2013) - Le recul des falaises crayeuses haut-normandes et les inondations par la mer en Manche centrale et orientale : de la quantification de l'aléa à la caractérisation des risques induits. Thèse de doctorat, Université de Caen, 408 p.

Mackay A., Battarbee R. W., Birks J., Oldfield F. (2005) - Global Change in the Holocene. Routledge, London, $480 \mathrm{p}$.

DOI : $10.1002 /$ jqs. 868

Mariotti J.F. (2018) - Les épaves racontent l'histoire de nos lacs, rivières et fleuves. Arcades, Numéro spécial, 44-47.

Mariotti J.F. (2019) - Taillebourg, un site portuaire du haut Moyen Âge. Arcades, Hors-Série, 34-37.

Moyat P. (2019) - Port-d'Envaux : découverte de trois bateaux mérovingiens au Priouté. Arcades, Hors-Série, 30-33. 
Nanson G.C., Croke J. C. (1992) - A genetic classification of floodplains. Geomorphology, 4 (6), 459-486.

Nicoll T., Hickin E. J. (2010) - Planform geometry and channel migration of confined meandering rivers on the Canadian Prairies. Geomorphology, 116 (1-2), 37-47.

DOI : 10.1016/j.geomorph.2009.10.005

Ollero A. (2010) - Channel changes and floodplain management in the meandering middle Ebro River Spain. Geomorphology, 117 (3), 247-260.

DOI : 10.1016/j.geomorph.2009.01.015

Pernin M. O. (1986) - Contribution à la méthodologie d'analyse de données longitudinales : exemple de la croissance chez l'être humain. Thèse de doctorat, Université Claude-Bernard de Lyon $1,264 \mathrm{p}$.

Perşoiu I., Rădoane M. (2011) - Spatial and temporal controls on historical channel responses - Study of an atypical case : Someşu Mic River, Romania. Earth Surface Processes and Landforms, 36 (10), 1391-1409.

DOI : $10.1002 /$ esp.2169

Pisŭt P. (2002) - Channel evolution of the pre-channelised Danube River in Bratislava, Slovakia (1712-1886). Earth Surface Processes and Landforms, 27 (4), 369-390.

DOI : 10.1002/esp.333

Pinard J. (1965) - La Charente, ses débits, ses crues et les marées de l'estuaire. Norois - Environnement, aménagement, société, 47, 357-363.

DOI : $10.3406 /$ noroi.1965.1533

Porter M. D., Massong T. M. (2002) - Analyzing changing river channel morphology using GIS for the Rio Grande Silvery Minnow Habitat Assessment, GIS/Spatial Analyses. Fishery and Aquatic Sciences, 433-446.

Rădoane M., Obreja F., Cristea I., Mihailă D. (2013) - Changes in the channel-bed level of the eastern Carpathian rivers: Climatic vs. Human control over the last 50 years. Geomorphology, 193, 91-111.

DOI : 10.1016/j.geomorph.2013.04.008

Renard R. (1993) - Les villes moyennes du fleuve Charente. Evolution historique et économique depuis l'Antiquité. Norois, 159, 413-429.

DOI : $10.3406 /$ noroi.1993.6495

Rieth E. (2010) - L'épave antique EP1-Taillebourg (CharenteMaritime). Un chaland de construction régionale réemployé en aménagement de rive. L'Archéo Thema - Revue d'archéologie et d'histoire, 6, 22-23.

Rinaldi M. (2003) - Recent channel adjustments in alluvial rivers of Tuscany, Central Italy. Earth Surface Processes and Landforms, 28 (6), 587-608.

DOI : $10.1002 /$ esp.464

Scorpio V., Aucelli P.P.C., Giano I., Pisano L., Robustelli G., Rosskopf C.M., Schiattarella M. (2015) - River channel adjustments in southern Italy over the past 150 years and implications for channel recovery. Geomorphology, 251, 77-90.

DOI : 10.1016/j/geomorph.2015.07.008

Scorpio V., Surian N., Cucato M., Dai Prá E., Zolezzi G., Comiti F. (2018) - Channel changes of the Adige River (Eastern Italian Alps) over the last 1000 years and identification of the historical fluvial corridor. Journal of Maps, 14 (2), 680-691.
DOI : $10.1080 / 17445647.2018 .1531074$

Segura-Beltrán F., Sanchis-Ibor C. (2013) - Assessment of channel changes in a Mediterranean ephemeral stream since in the early twentieth century. The Rambla de Cervera, Eastern Spain. Geomorphology, 201, 199-214.

DOI : 10.1016/j.geomorph.2013.06.021

Serlet A. J., Gurnell A. M., Zolezzi G., Wharton G., Belleudy P., Jourdain C. (2018) - Biomorphodynamics of alternate bars in a channelized, regulated river: an integrated historical and modelling analysis. Earth Surface Processes and Landforms, 43 (9), 1739-1756.

DOI : $10.1002 /$ esp.4349

Slowik M. (2013) - Transformation of a lowland river from a meandering and multi-channel pattern into an artificial canal: Retracing a path of river channel changes (the Middle Obra River, W Poland). Regional Environmental Change, 13 (5), 1287-1299. DOI : 10.1007/s10113-013-0453-3

Slowik M. (2015) - Is history of rivers important in restoration projects? The example of human impact on a lowland river valley (the Obra River, Poland). Geomorphology, 251, 51-63.

DOI : 10.1016/j.geomorph.2015.05.031

Slowik M., Dezsõ J., Kovács J., Galka M. (2020) - The formation of low-energy meanders in loess landscapes (Transdanubia, central Europe). Global and Planetary Change, 184.

DOI : 10.1016/j.gloplacha.2019.103071

Surian N., Ziliani L., Comiti F., Lenzi M.A., Mao L. (2009) Channel adjustments and alteration of sediment fluxes in gravelbed rivers of North-Eastern Italy: Potentials and limitations for channel recovery. River Research and Applications, 25 (5), 551567.

DOI : $10.1002 /$ rra.1231

Swanson B.J., Meyer G.A., Coonrod J.E. (2010) - Historical channel narrowing along the Rio Grande near Albuquerque, New Mexico in response to peak discharge reductions and engineering: magnitude and uncertainty of change from air photo measurements. Earth Surface Processes and Landforms, 36 (7), 885-900.

DOI : $10.1002 /$ esp.2119

Thieler R. E., Danforth W. W. (1994a) - Historical shoreline mapping I: Improving techniques and reducing positioning errors. Journal of Coastal Research, 10 (3), 549-563.

Thieler R. E., Danforth W. W. (1994b) - Historical shoreline mapping II: Application of the digital shoreline mapping and analysis systems (DSMS/DSAS) to shoreline change mapping in Puerto Rico. Journal of Coastal Research, 10 (3), 600-620.

Tiegs S., Pohl M. (2005) - Planform channel dynamics of the lower Colorado River: 1976-2000. Geomorphology, 69 (1), 14-27.

DOI : 10.1016/j.geomorph.2004.12.002

Tillard E. (1996) - Analyse exploratoire de tableaux de données indicés dans le temps en épidémiologie, en écologie et en agronomie. Mémoire de DEA, Université Claude Bernard de Lyon 1, $32 \mathrm{p}$.

Toone J., Rice S. P., Piégay H. (2014) - Spatial discontinuity and temporal evolution of channel morphology along a mixed bedrock-alluvial river, upper Drôme River, southeast France: Contingent responses to external and internal controls. Geomorphology, 205, 5-16. 
DOI : 10.1016/j.geomorph.2012.05.033

Uribelarrea del Val D., Pérez-González A., Benito G. (2003) Channel changes in the Jarama and Tagus Rivers (central Spain) over the past 500 years. Quaternary Science Reviews, 22 (20), 2209-2221.

DOI : $10.1016 / S 0277-3791(03) 00153-7$

Vayssière A. (2018) - Trajectoires et processus fluviaux dans la moyenne vallée du Cher du Tardiglaciaire à la période actuelle : métamorphose fluviale, réponses aux forçages sociétaux et ajustements des chenaux et des bras morts. Thèse de doctorat, Université Paris I Panthéon Sorbonne, 467 p.

Vayssière A., Rué M., Recq C., Gardère P., Bozsó E., Castanet C., Virmoux C., Gautier E. (2019) - Lateglacial changes in river morphologies of northwestern Europe : An example of a smooth response to climate forcing (Cher River, France). Geomorphology, 342, 20-36.

DOI : 10.1016/j.geomorph.2019.05.019

Winterbottom S.J. (2000) - Medium and short-term channel planform changes on the Rivers Tay and Tummel, Scotland. Geomorphology, 34 (3-4), 195-208.

DOI : 10.1016/S0169-555X(00)000007-6

Zawiejska J., Wyzga B. (2010) - Twentieth-century channel change on the Dunajec River, southern Poland: Patterns, causes and controls. Geomorphology, 117 (3), 234-246.

DOI : 10.1016/j.geomorph.2009.01.014

Ziliani L., Surian N. (2012) - Evolutionary trajectory of channel morphology and controlling factors in a large gravel-bed river. Geomorphology, 173-174, 104-117.

DOI : 10.1016/j.geomorph.2012.06.001

\section{Abridged English version}

The study of historical channel changes is a fundamental knowledge for an efficient river management and restoration. Although many studies have analyzed the channel changes on European great rivers or mountain rivers over the last two centuries, researches have rarely focused on the historic evolution of low-energy and low mobile rivers.

This study documents the historic evolution of the middle Charente River (fluvial section between Angoulême and Saintes) (fig.1) from 1866 to 2017. The study area is part of the low-energy rivers of the European Atlantic lowlands (Candel, 2020) with a stream power lower than $10 \mathrm{~W} \cdot \mathrm{m}^{-2}$, a very low mean slope of $0.86 \%$ and a mean interannual discharge of $140 \mathrm{~m}^{3} \cdot \mathrm{s}^{-1}$. Banks cohesiveness is high because of their silt content. The floodplain is characterised by anastomosing fluvial landscapes (multi-channels and vegetated islands) in the upstream river reach (section between Angoulême and Cognac) and meandering fluvial landscapes in its downstream river reach (section between Cognac and Saintes). The study segment has been subjected to high anthropogenic pressures since at least the High Middle Ages (Dumont et Mariotti, 2013) and was enhanced during the $19^{\text {th }}$ century to facilitate navigation and terrestrial transportation, to ensure the using of the water's driving force (water mills and paper mills), to maintain the local people (fishing dams and agro-pastoral uses) and to allow for flood protection.

This paper aims to analyze the historic evolution of the middle Charente River over the last 250 years and to adapt the geomorphometric method applied so far on middle and high energy rivers where the fluvial adjustments are difficult to demonstrate due to closeness between speed of changes and the error margin associated with the quantitative method. The method is based on a multi-temporal quantitative approach conducted on four historical maps (1866, 1899, 1972 and 2017) among the initial corpus including nineteen maps of the Charente River. Geohistorical data was integrated in a GIS environment (Qgis 2.18.14 and PostGIS database): historical maps were georeferenced onto a base map (SCAN 25) using projection Lambert-93, the alluvial plain was digitized and channel planforms were digitized from the four maps. The alluvial plain was then subdivided into sectors $500 \mathrm{~m}$ in length following the Thiessen's polygonalisation method (Albert and Piégay, 2011; David et al., 2016): 181 sectors were generated along the study linear. Sixteen geomorphometrical parameters were measured or calculated at the sector scale for each date on the channel planforms digitized earlier (fig. 6). Geomorphometrical data generated at the sector scale are then subject to factor analysis by Principal Component Analysis (PCA) and Hierarchical Cluster Analysis (HCA) using the software R 3.5.0. Three classes were adopted. The characteristics of classes depend on the presence, size, perimeter and form of the islands. HCA results for each date are subject to automatic detection of spatial ruptures using the software R 3.5.0.

The results enable to propose a segmentation of the study reach into four homogeneous zones defined by homogeneous evolutionary trajectory: zone Z1 (Angoulême to Châteauneuf-sur-Charente), zone Z2 (Angeac-Charente to Bourg-Charente), zone Z3 (Saint-Brice to Merpins) and zone Z4 (Saint-Laurent-de-Cognac to Saintes). It also shows four steps of evolution of the Charente River: (i) a global stabilization of the study reach on the period 1866-2017; (ii) a simplification of the fluvial mosaic on the upstream river between 1866 and 1899; (iii) the complexity of the fluvial mosaic between 1899 and 1972 with the apparition of new islands; and (iv) the transformation of the single channel sinuous sectors to anastomosing sectors (high and elongated islands) in particular downstream river between 1972 and 2017.

This study shows the stability of the channels and islands of Charente River. This stability is interpreted as a combination of the very lowenergy fluvial system and the banks cohesion. This stability seems only to be broken by the human interventions over the last two centuries. At a local scale, most of the Charente River evolutions are controlled by the navigation and the maintenance of the channel and islands. The lack of geohistorical data and the limitations of statistic method do not enable to qualify and quantify the part of other anthropogenic controlling factors in the fluvial system managed essentially for the navigation.

This first results show that the middle Charente River is a unique fluvial system. The researches of Lewin (2010) and Brown et al. (2018) concluded that the anastomosing rivers are relics of fluvial landscapes weakly modified by human interventions due to a combination of geological causes and social remoteness. This affirmation must be qualified because the Charente River has been subjected to high anthropogenic pressures since the High Middle Ages, however has preserved anastomosed patterns. This contradiction can be explained as the combination of an adjustment of the fluvial system morphology and an adjustment of installations related to specificities associated with a low-energy river. 